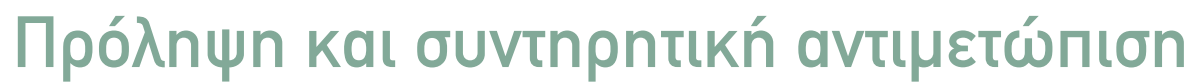

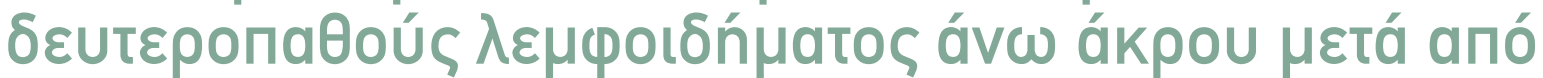

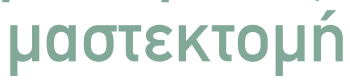

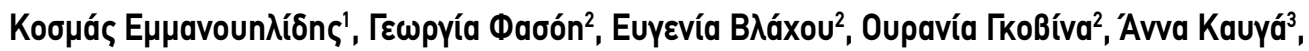

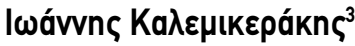

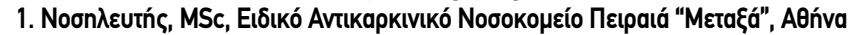

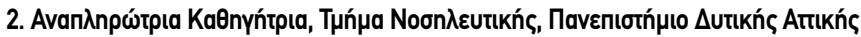

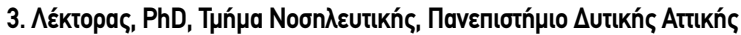

\section{ПEPIAHWH}

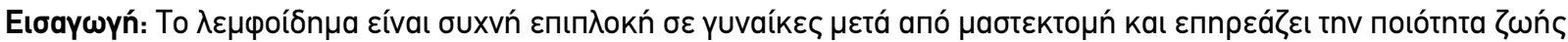

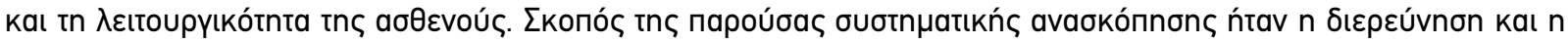

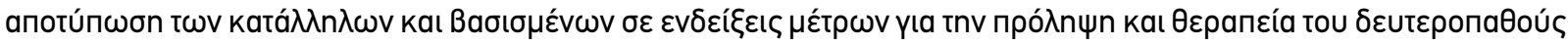

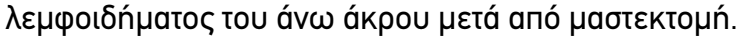

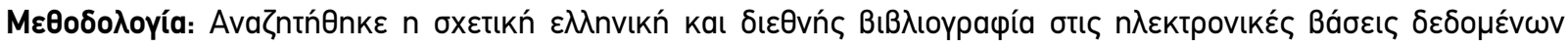

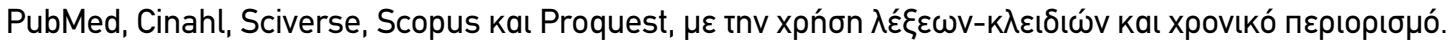

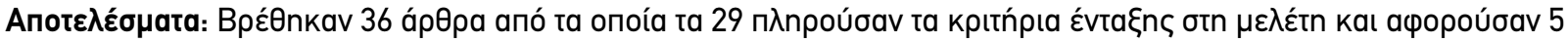

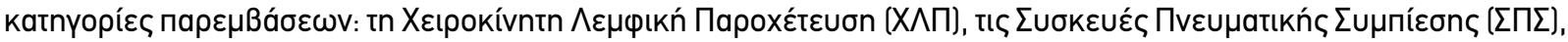

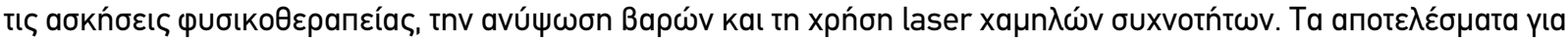

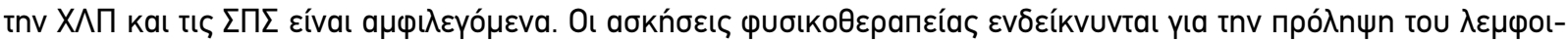

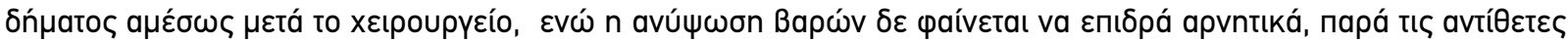

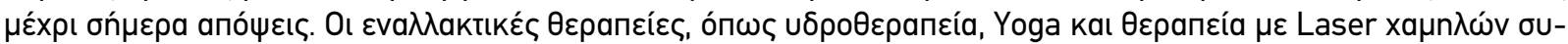

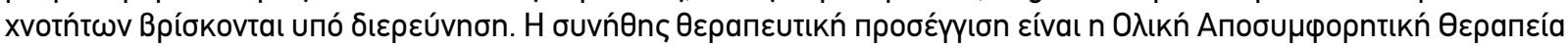

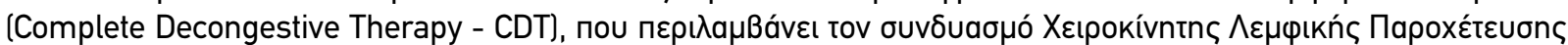

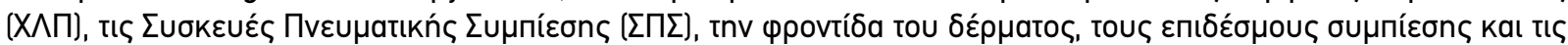

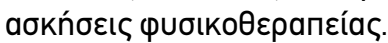

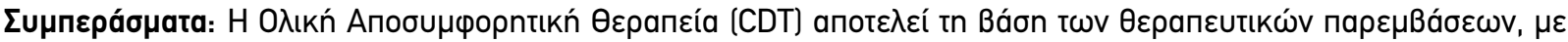

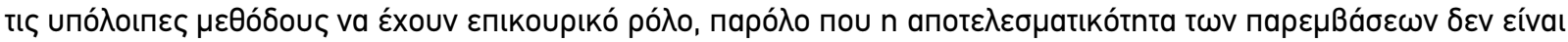

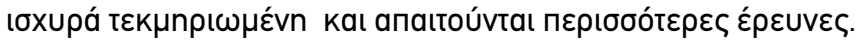

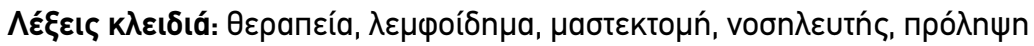




\section{EPEYNHTIKH EPTAEIA}

\section{BAEIKA EHMEIA}

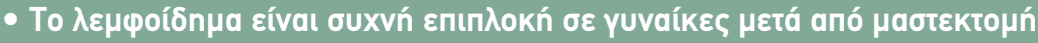

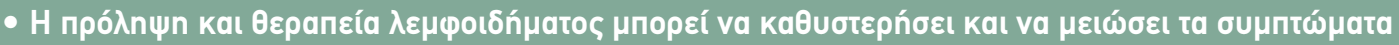

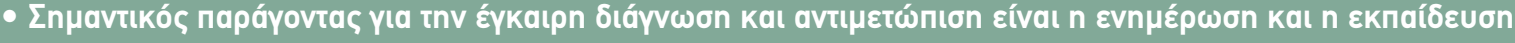

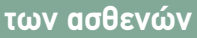

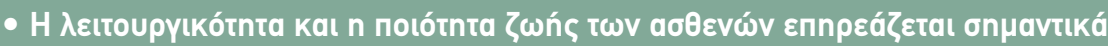

\section{EIIAГQГH}

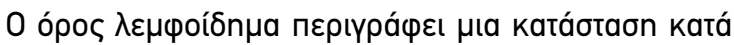

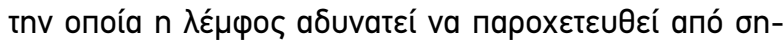

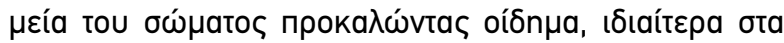

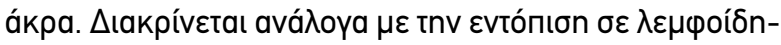

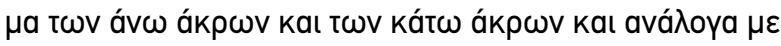

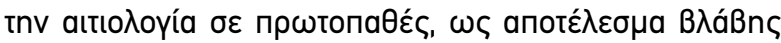

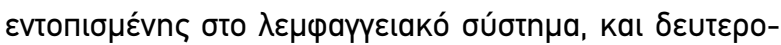

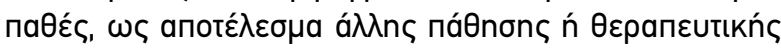

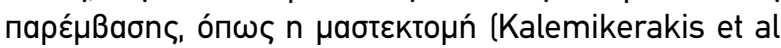
2012).

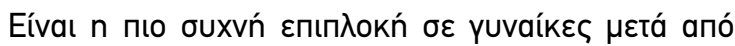

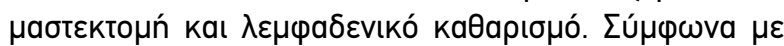

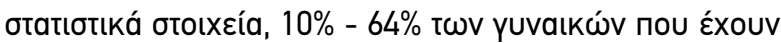

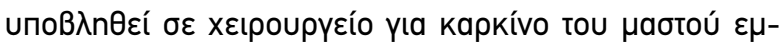

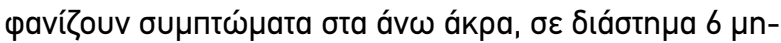

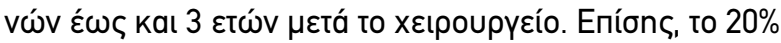

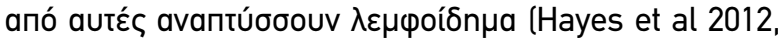
Paskett et al 2012).

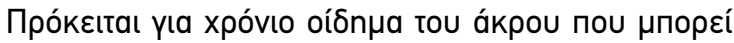

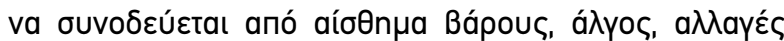

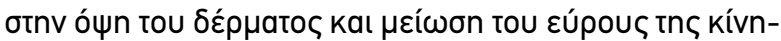

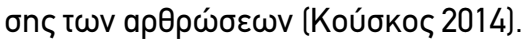

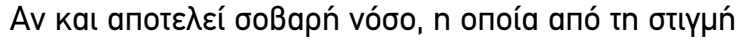

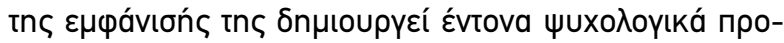

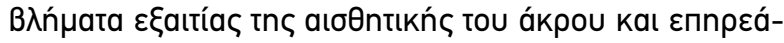

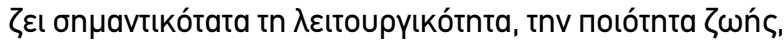

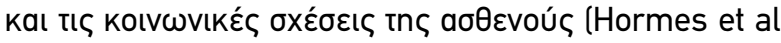

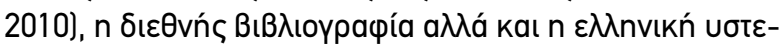

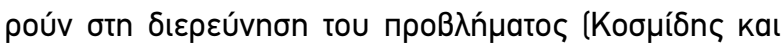
ouv 2009, Kalemikerakis et al 2012).

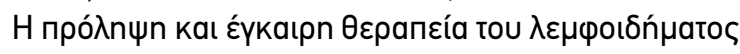
$\mu п$

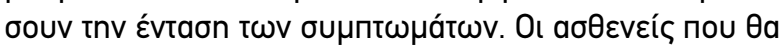

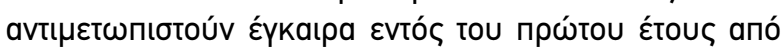

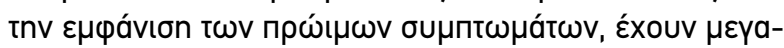

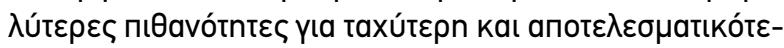

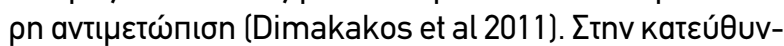

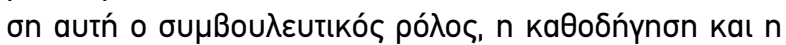

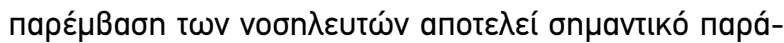

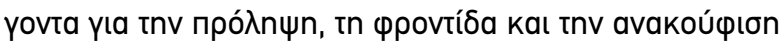

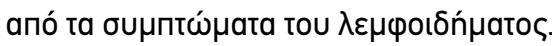

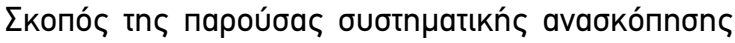

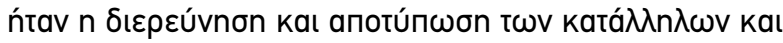

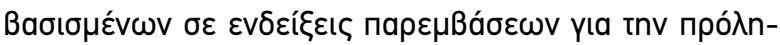

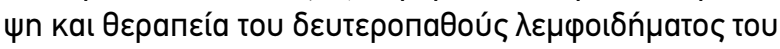

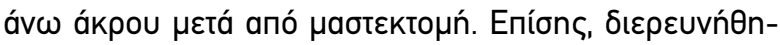

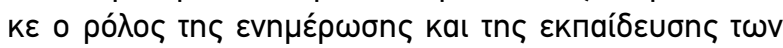

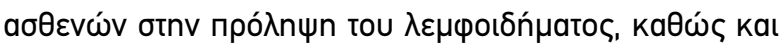

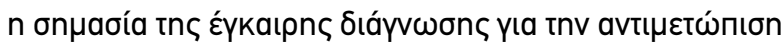
tnç vóoou.

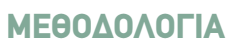

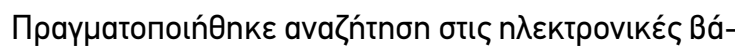

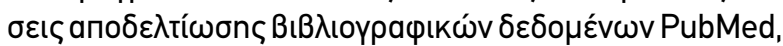
Cinahl, Sciverse, Scopus kal Proquest. Oı $\lambda \varepsilon ́ \xi \varepsilon ı \varsigma ~ k \lambda \varepsilon l-$

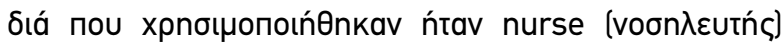

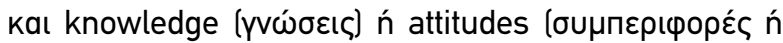

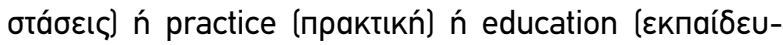

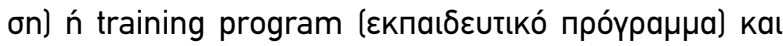

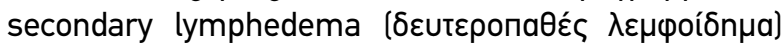

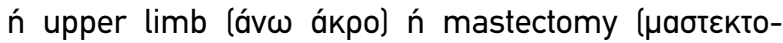

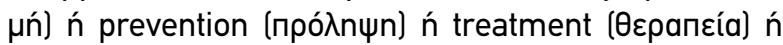

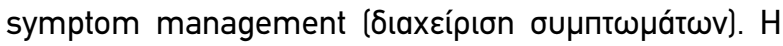

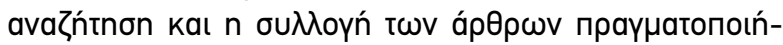

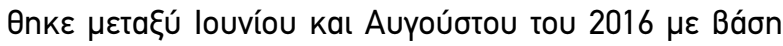

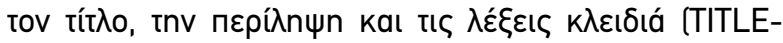

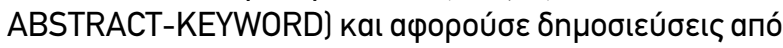

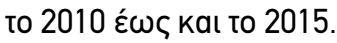

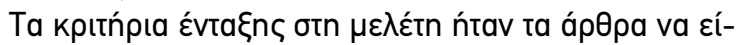

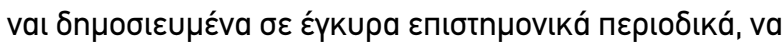

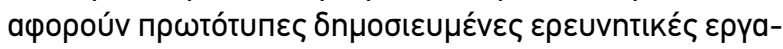

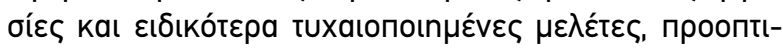

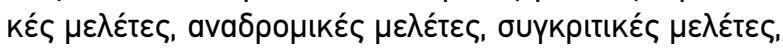

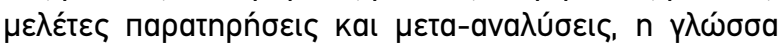

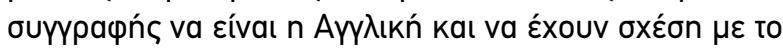

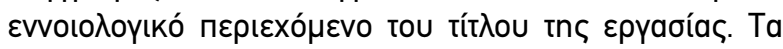

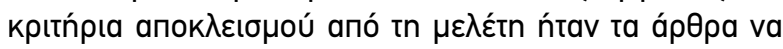

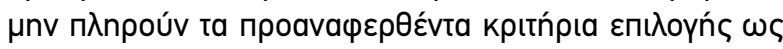

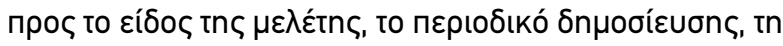

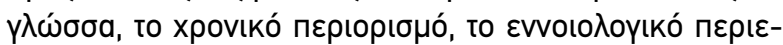

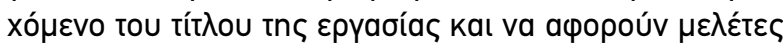

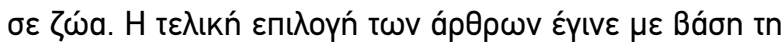

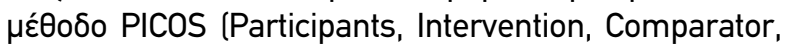




\section{EPEYNHTIKH EPГAIIA}

Outcomes, Study design) (nívakas 1).

\section{AПOTEАEЕMATA}

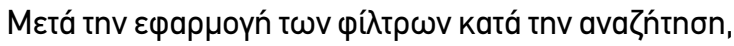

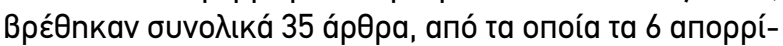

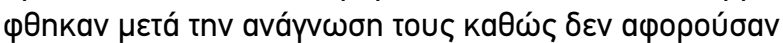

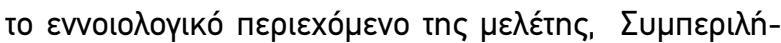

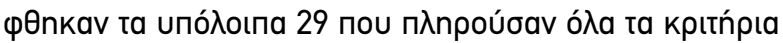

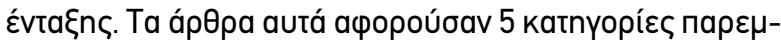

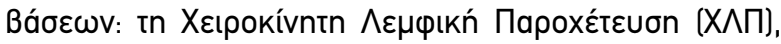

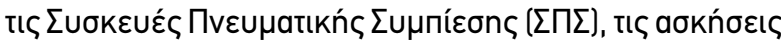

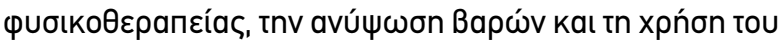

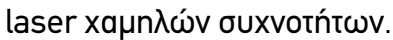

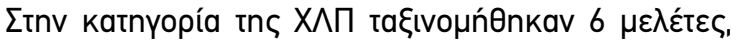

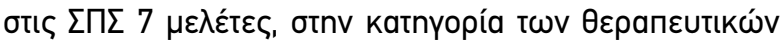

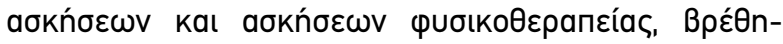

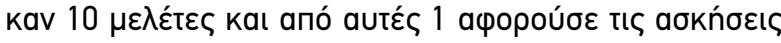

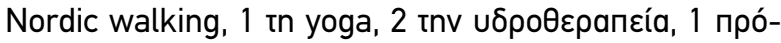

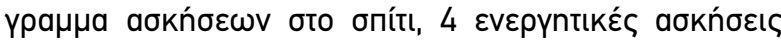

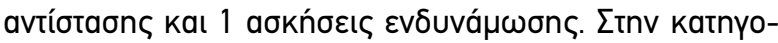

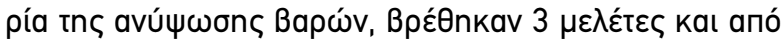

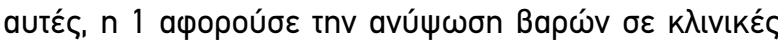

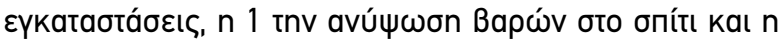

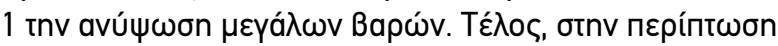

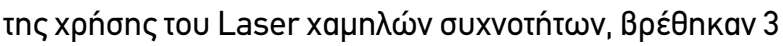

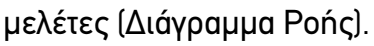

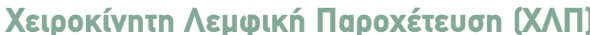

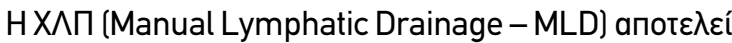

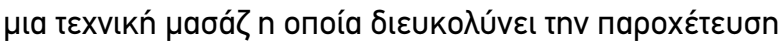

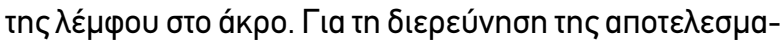

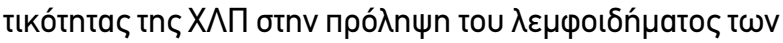

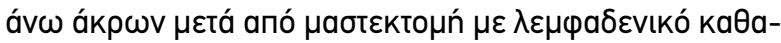

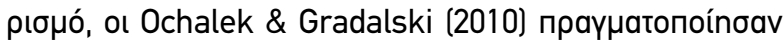

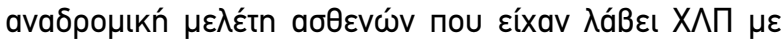

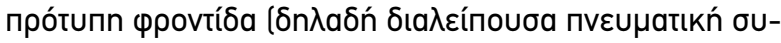

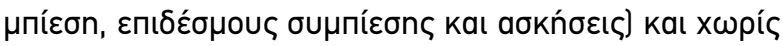

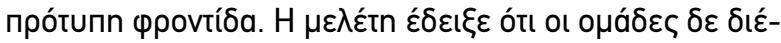

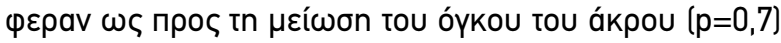

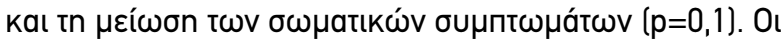

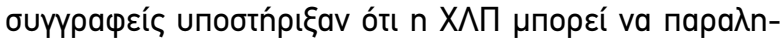

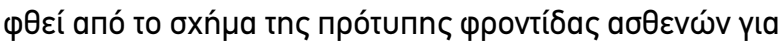

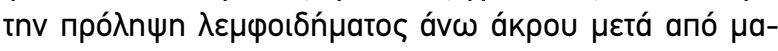

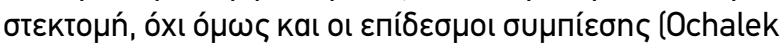
\& Gradalski 2010).

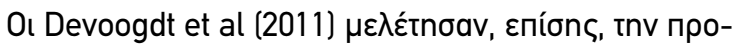

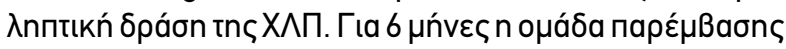

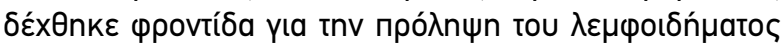

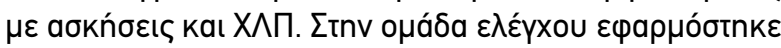

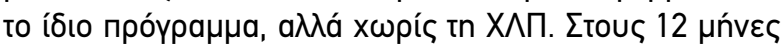

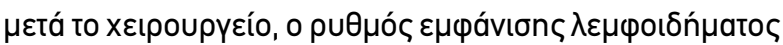

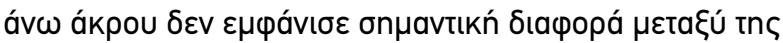

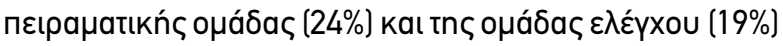

$(\mathrm{p}=0,5)$ (Devoogdt et al 2011).

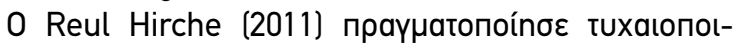

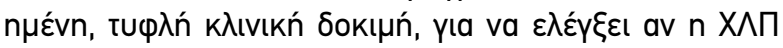

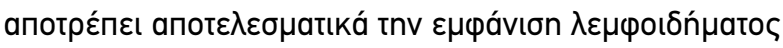

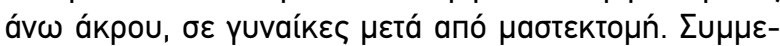

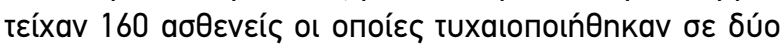

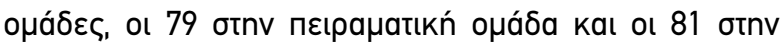

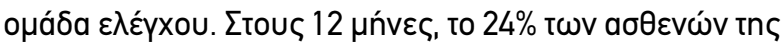

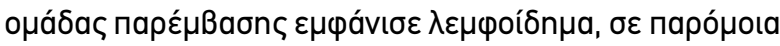

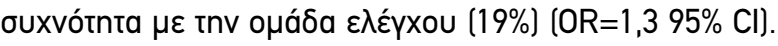

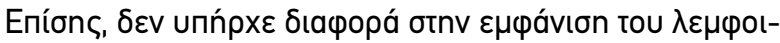

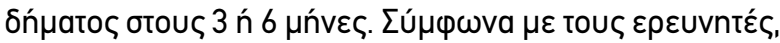

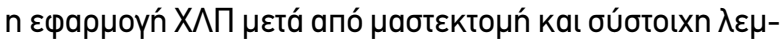

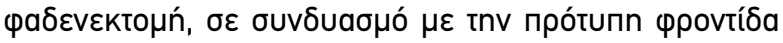

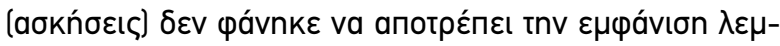

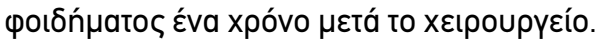

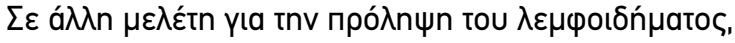

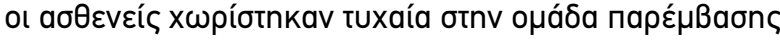

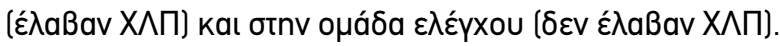

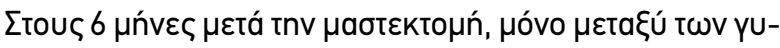

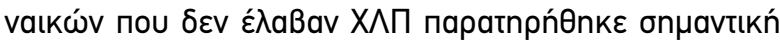

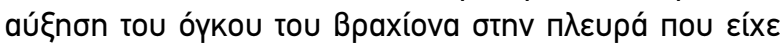

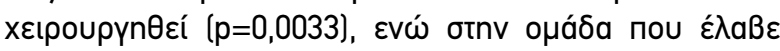

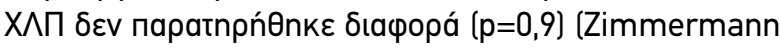
et al 2012).

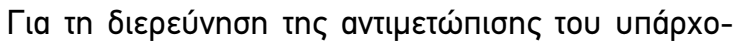

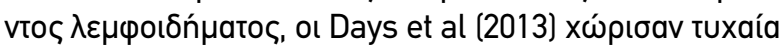

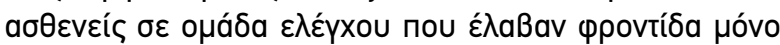

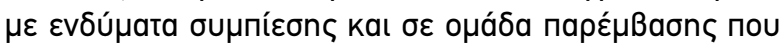

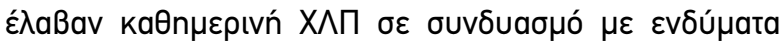

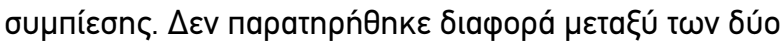

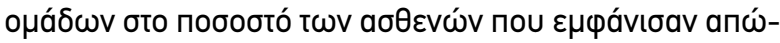

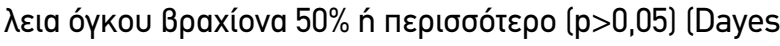
et al 2013).

Eníons, ol de Oliveira et al (2014) праүuatonoínoav

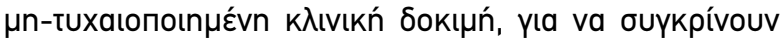

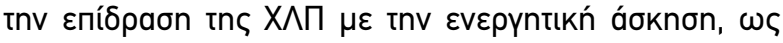

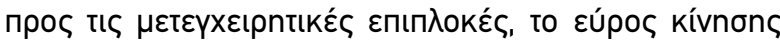

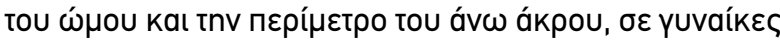

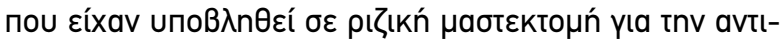

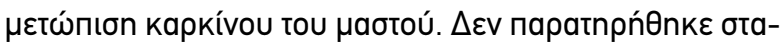

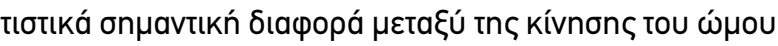

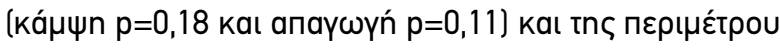
tou ákpou $(p=0,58)$ (De Oliveira et al 2014).

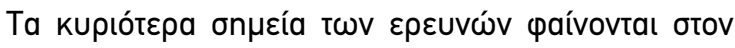
пívaka 2.

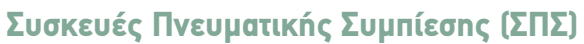

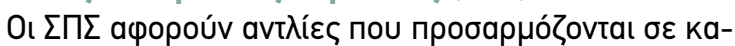

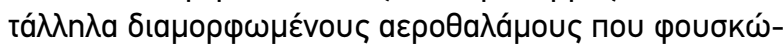

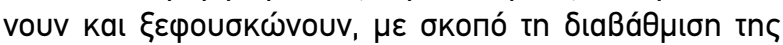

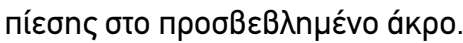

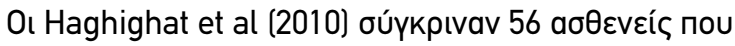




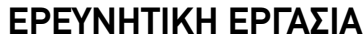

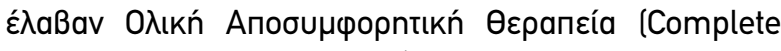

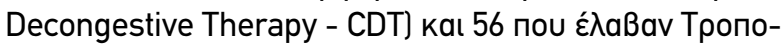

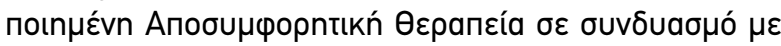

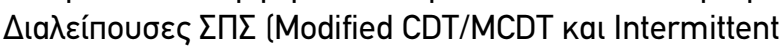

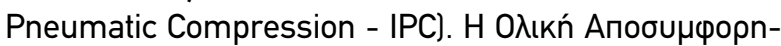

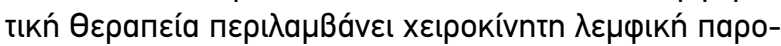

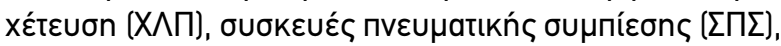

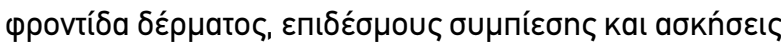

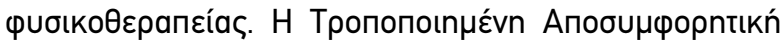

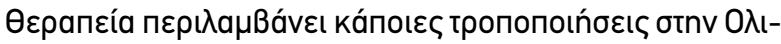

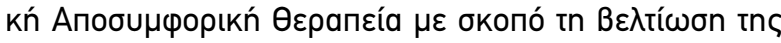

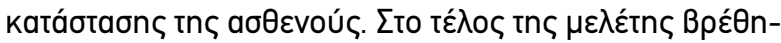

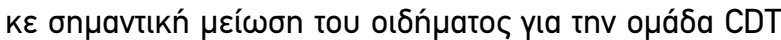
Évavtı tnc MCDT kaı IPC ( $p=0,036)$ (Haghighat et al 2010).

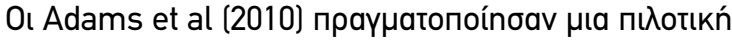

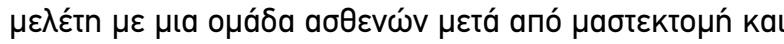

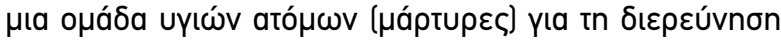

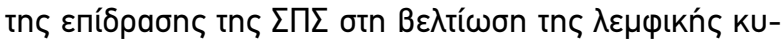

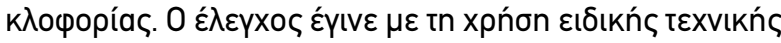

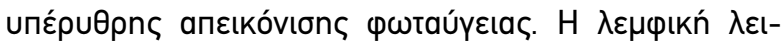

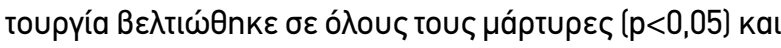

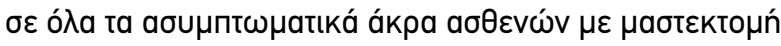

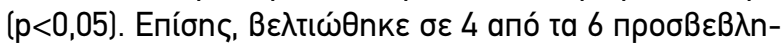

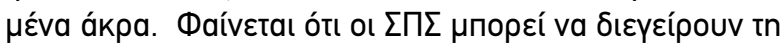

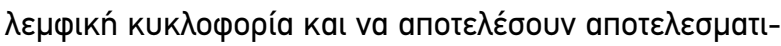

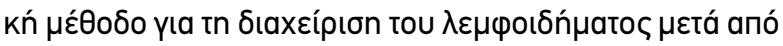

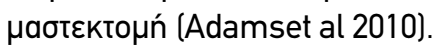

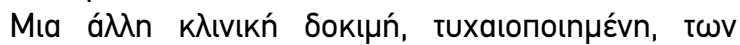

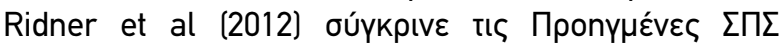
(Advanced Pneumatic Compression Devices - APCD) $\sigma \varepsilon$

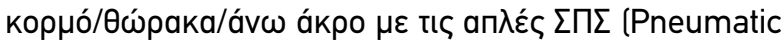

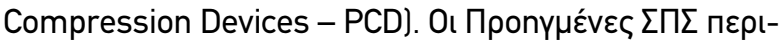

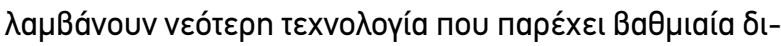

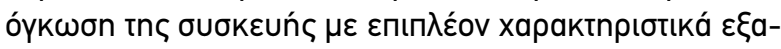

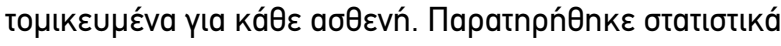

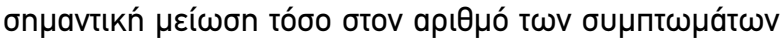

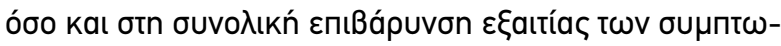

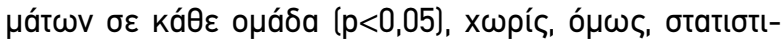

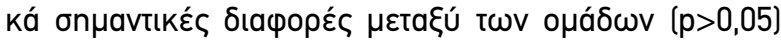

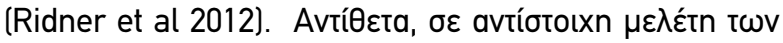

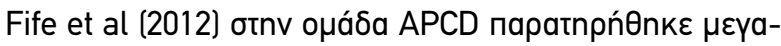

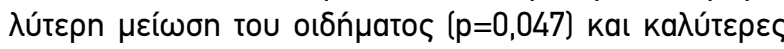

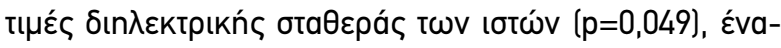

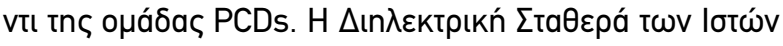

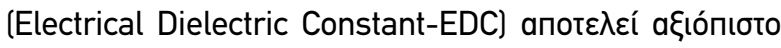

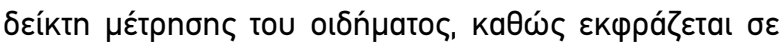

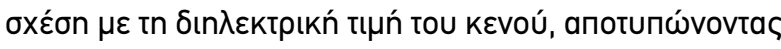

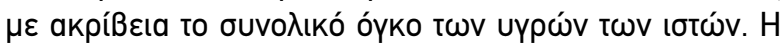

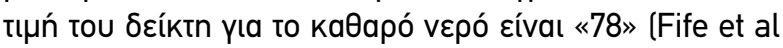
2012).

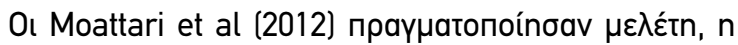

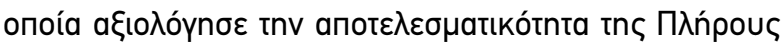

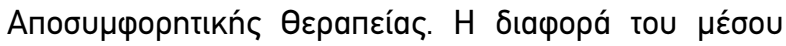

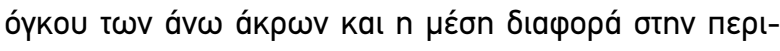

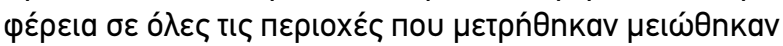

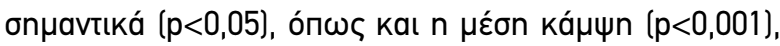

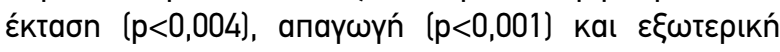

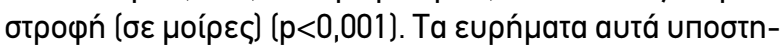

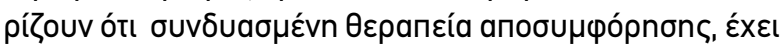

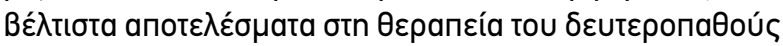

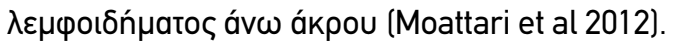

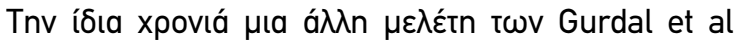

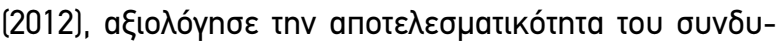

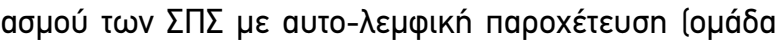

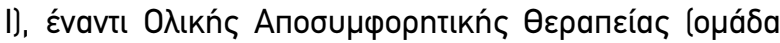

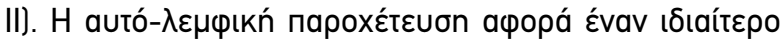

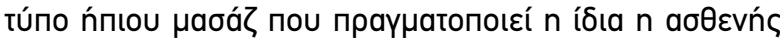

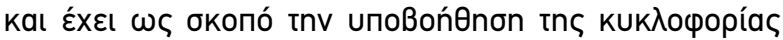

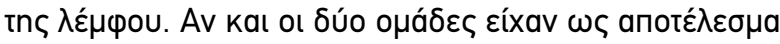

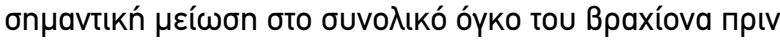

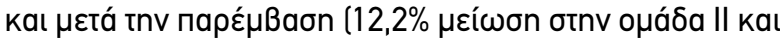

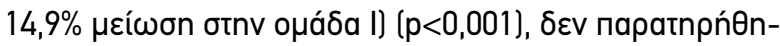

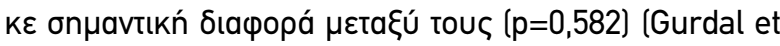
al 2012)

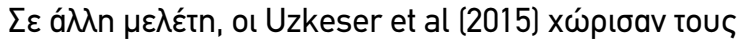

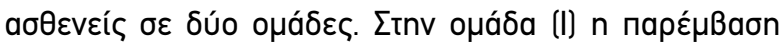

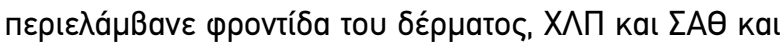

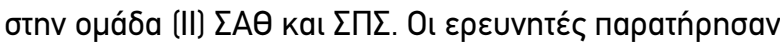

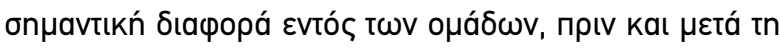

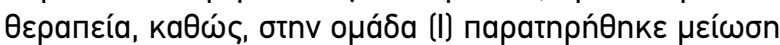

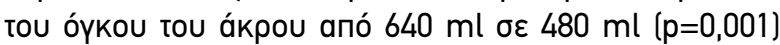

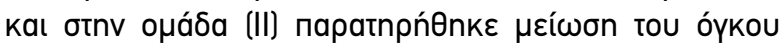

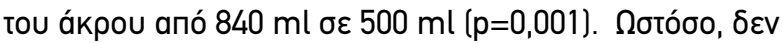

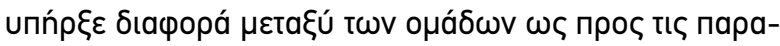

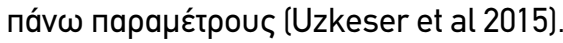

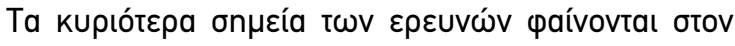
пívaka 3.

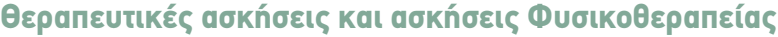

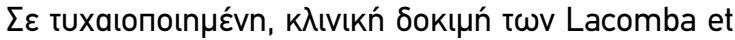

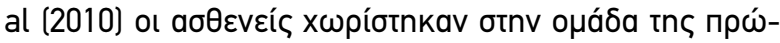

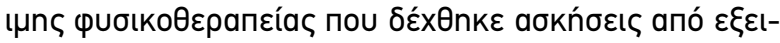

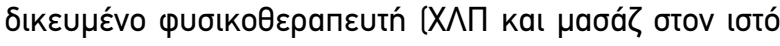

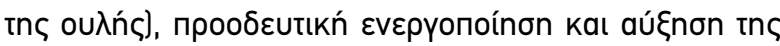

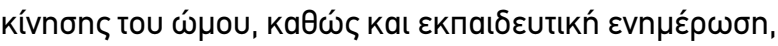

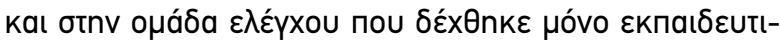

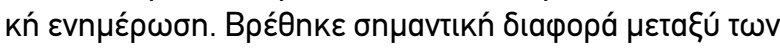

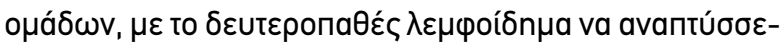

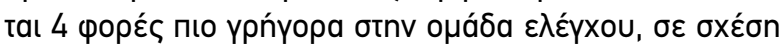

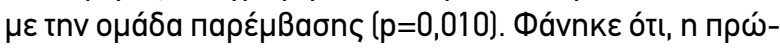

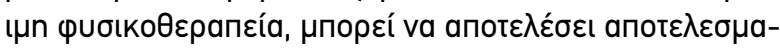

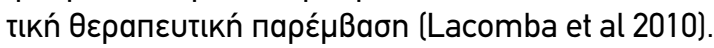

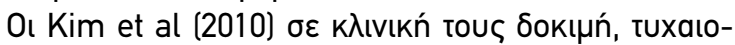

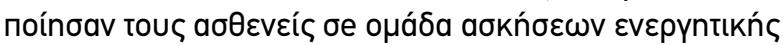

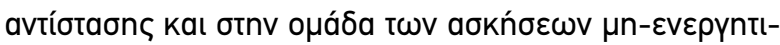

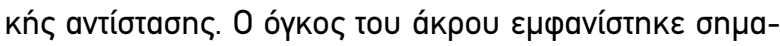




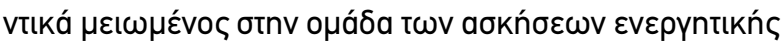

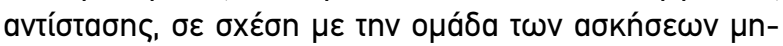

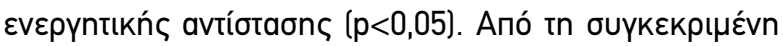

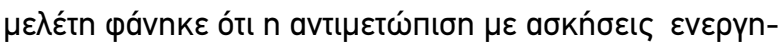

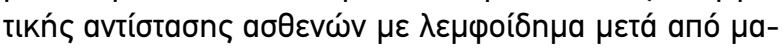

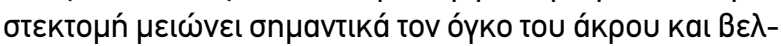

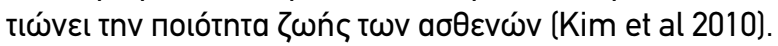

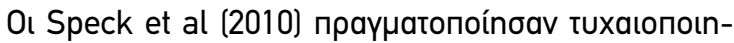

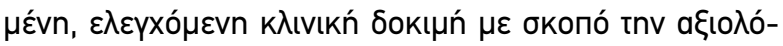

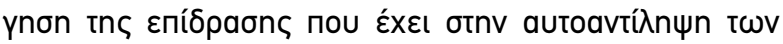

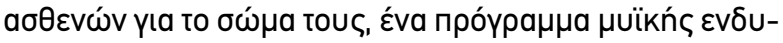

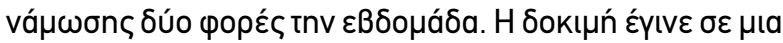

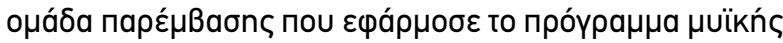

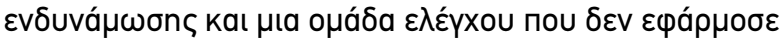

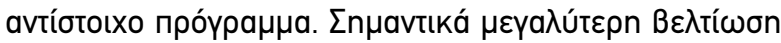

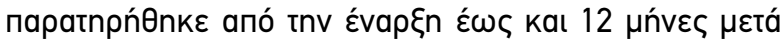

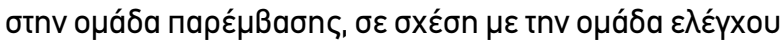

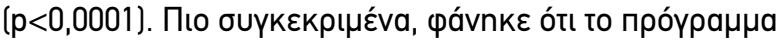

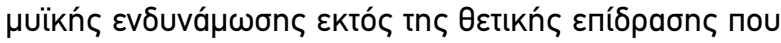

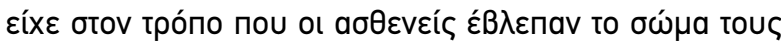

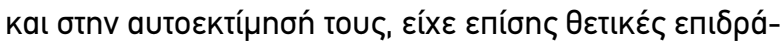

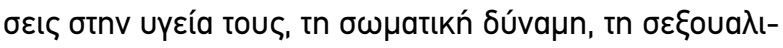

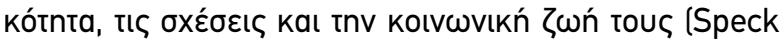
et al 2010).

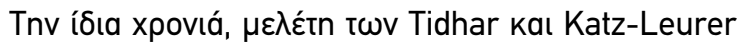

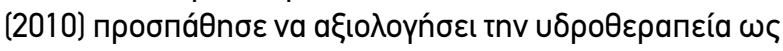

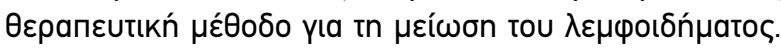

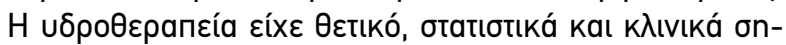

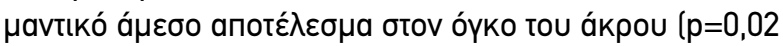

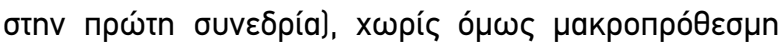

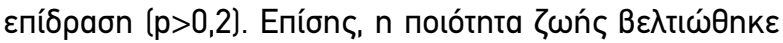

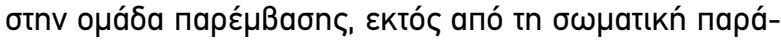

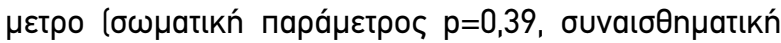

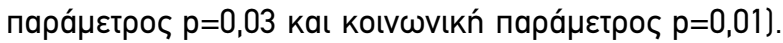

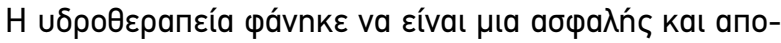

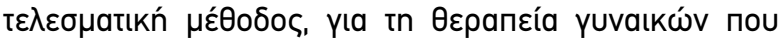

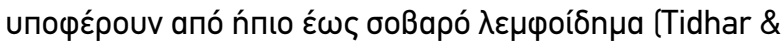
Katz-Leurer 2010).

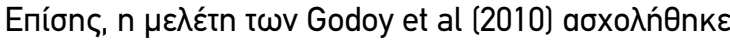

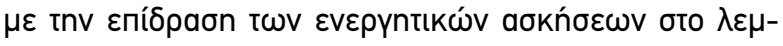

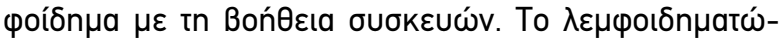

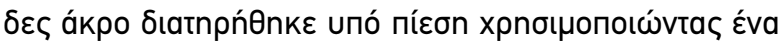

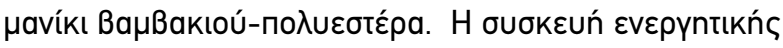

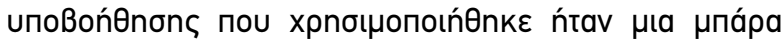

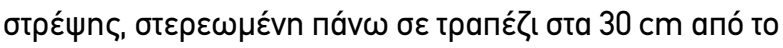

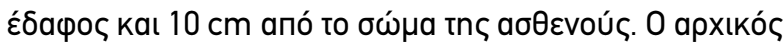

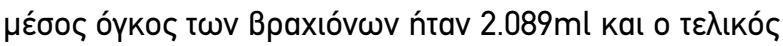

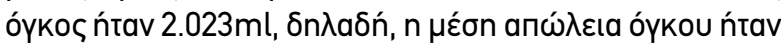

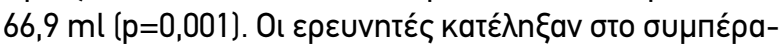

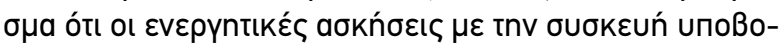

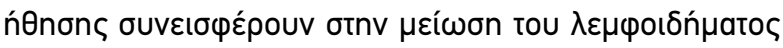
tou ákpou (Godoy et al 2010).

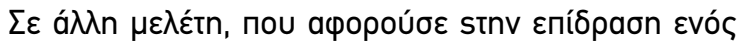

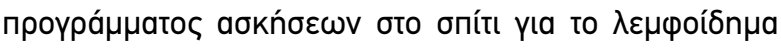

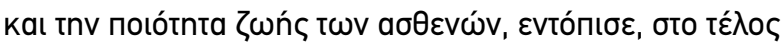

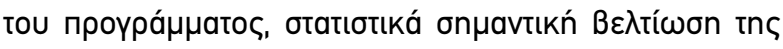

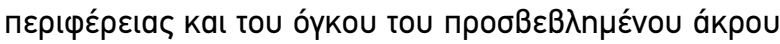

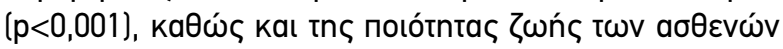
$(\mathrm{p}<0,001)$ (Gautam et al 2011).

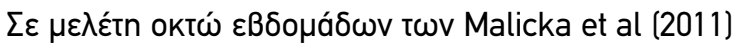

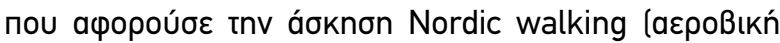

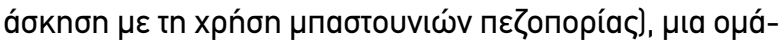

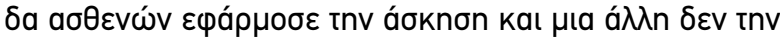

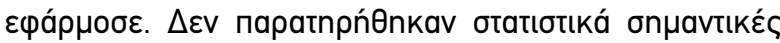

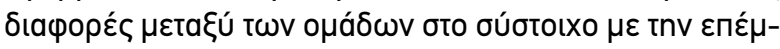

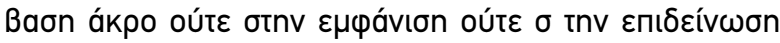

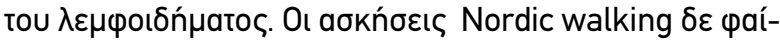

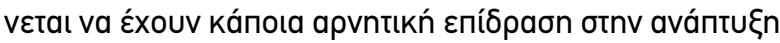

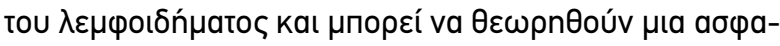

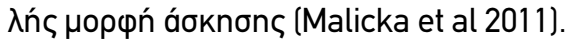

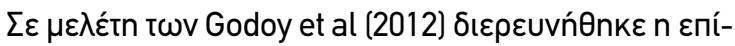

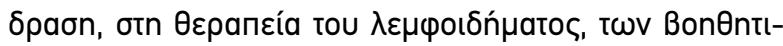

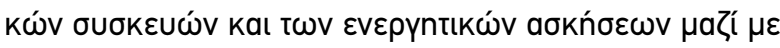

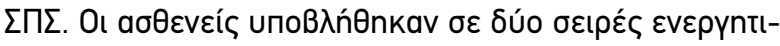

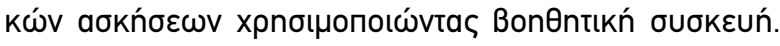

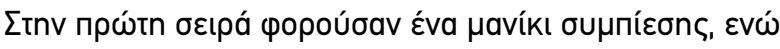

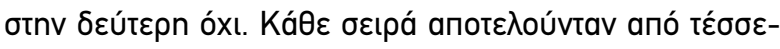

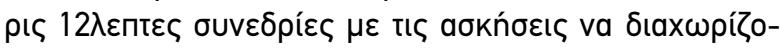

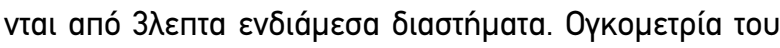

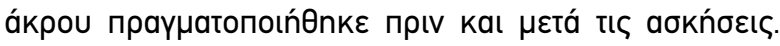

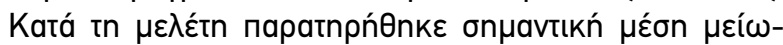

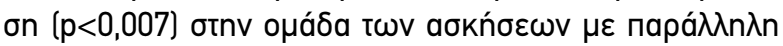

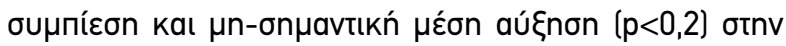

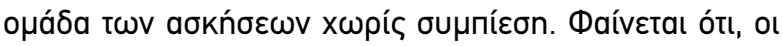

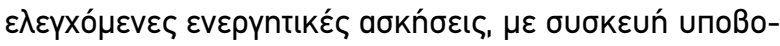

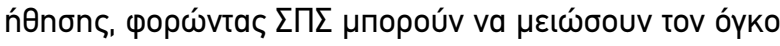

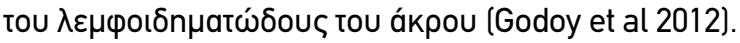

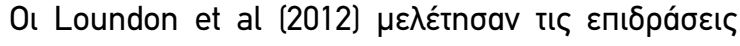

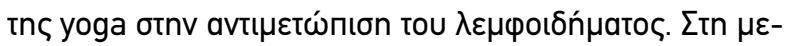

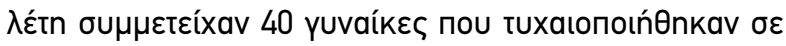

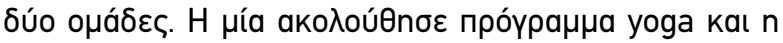

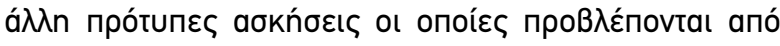

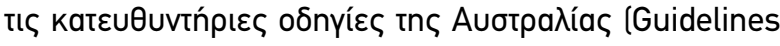
of The Australasian Lymphology Association and

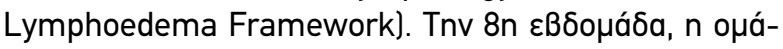

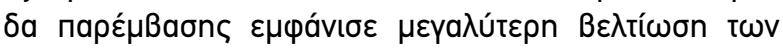

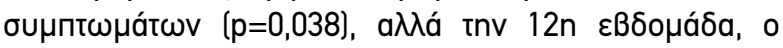

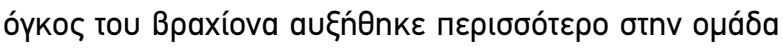

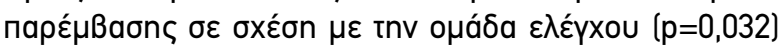
(Loundon et al 2012).

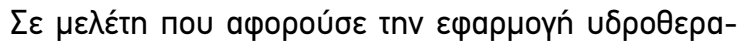

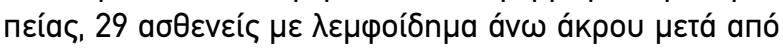

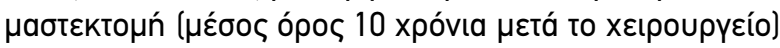

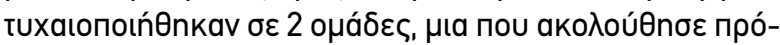

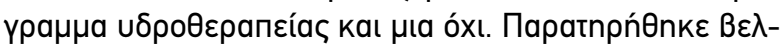

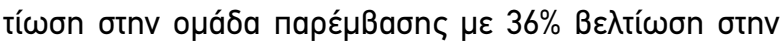




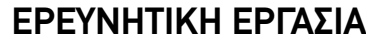

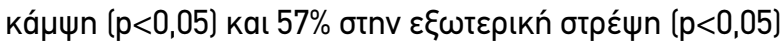

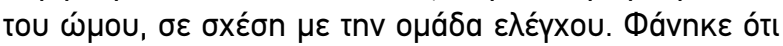

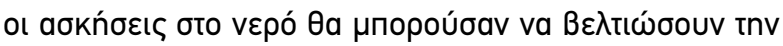
kívnon tou $\omega ́ \mu$ ou (Johansson et al 2013).

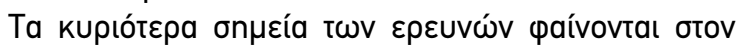
пívaka 4.

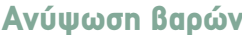

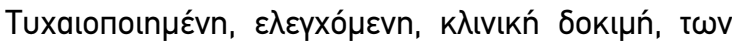

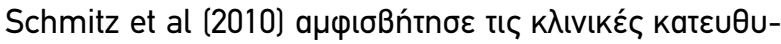

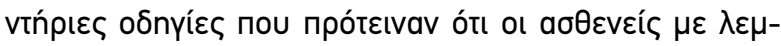

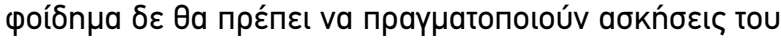

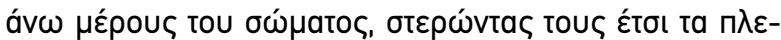

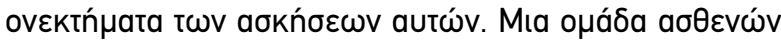

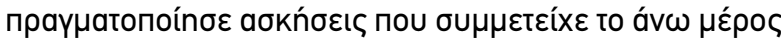

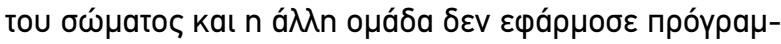

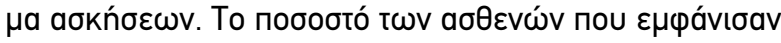

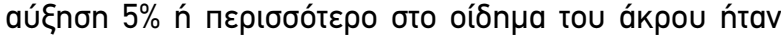

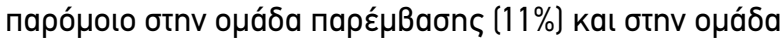

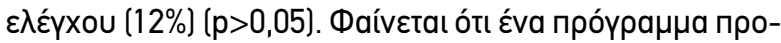

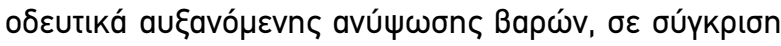

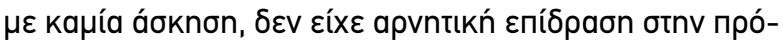
$\lambda n \psi n$ tou $\lambda \varepsilon \mu \varphi$ oاठ́n $\mu$ atos (Schmitz et al 2010).

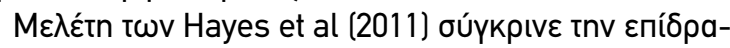

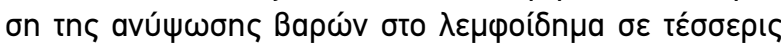
о

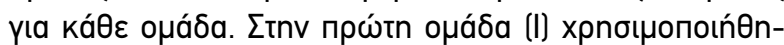

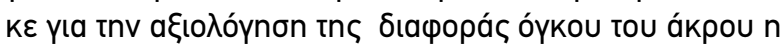

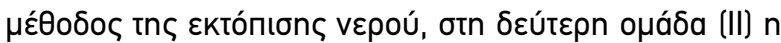

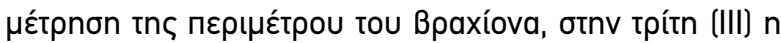

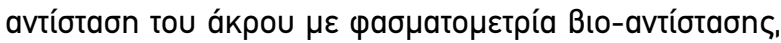

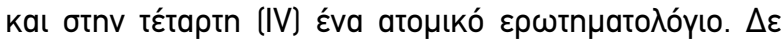

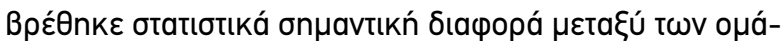

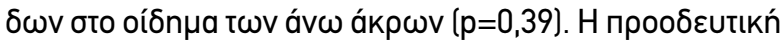

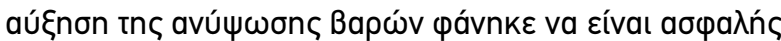

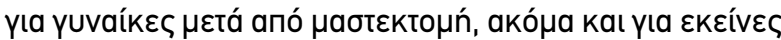

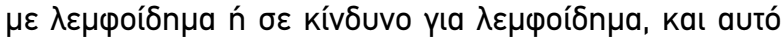

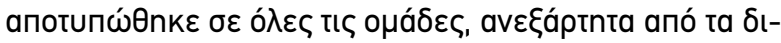

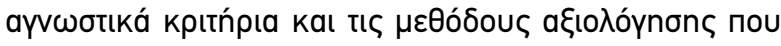
xpnoumonoıńӨnkav (Hayes et al 2011).

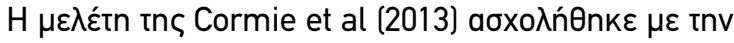

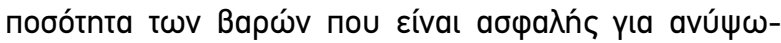

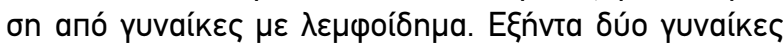

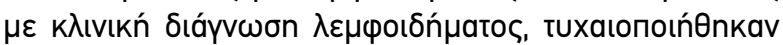

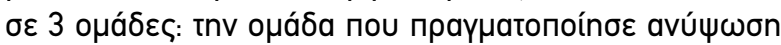

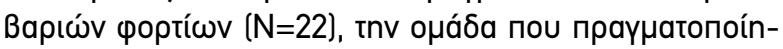

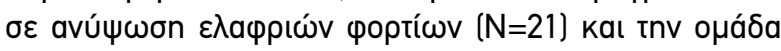

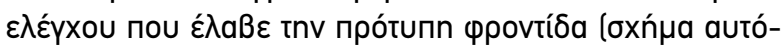

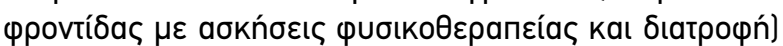

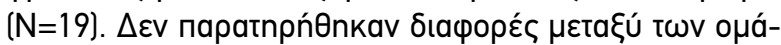

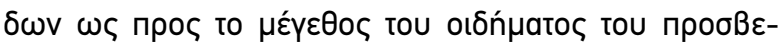

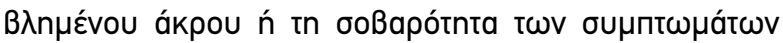

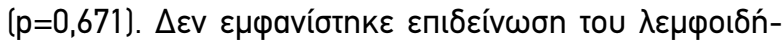

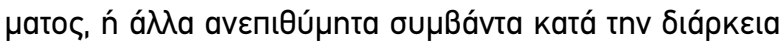
tn

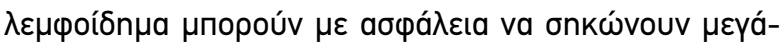

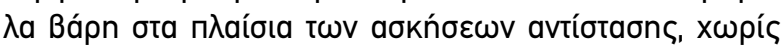

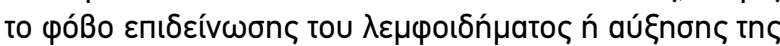

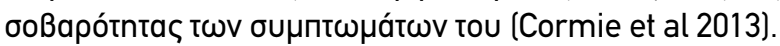

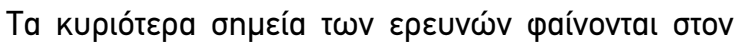
пívaka 5.

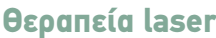

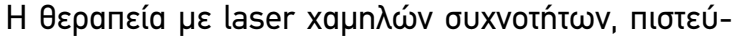

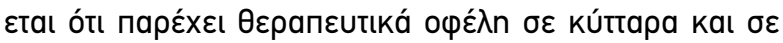

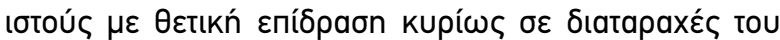

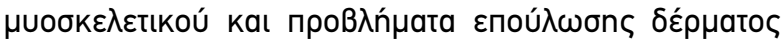

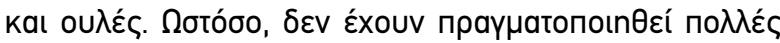

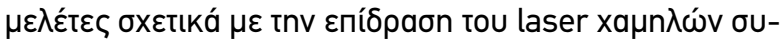

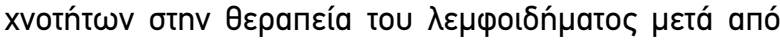

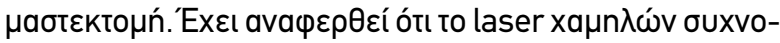

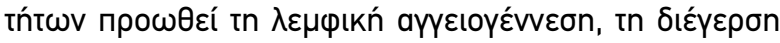

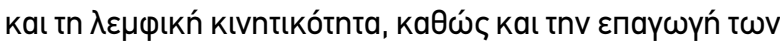

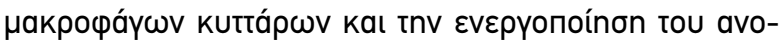

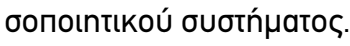

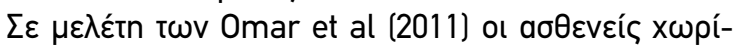

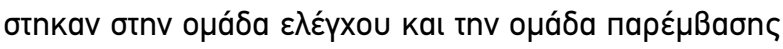

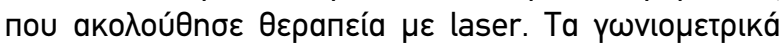

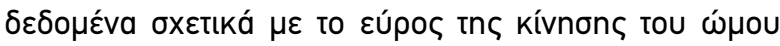

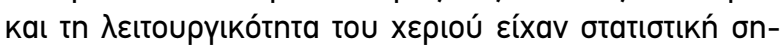

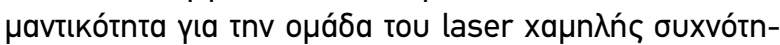

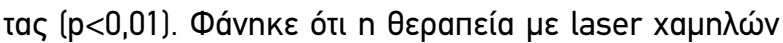

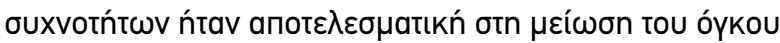

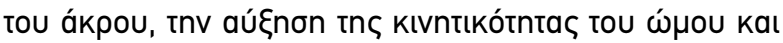

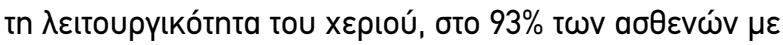

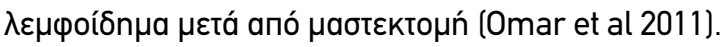

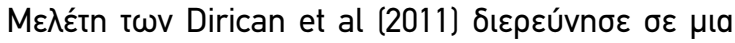

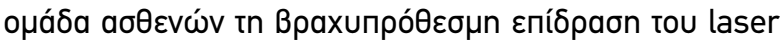

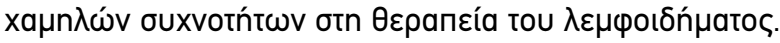

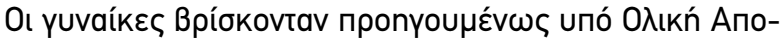

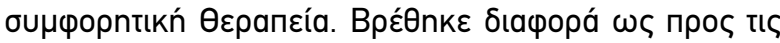

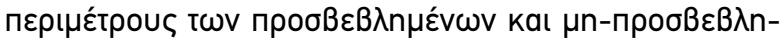

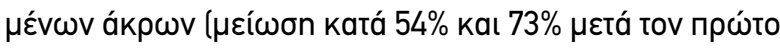

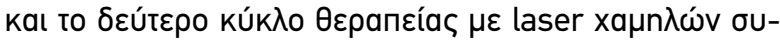

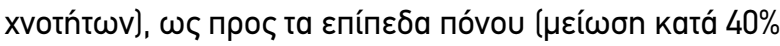

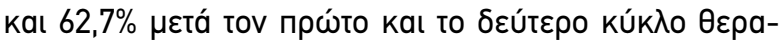

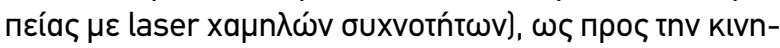

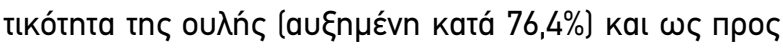

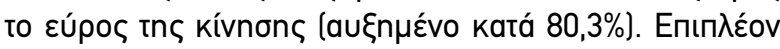

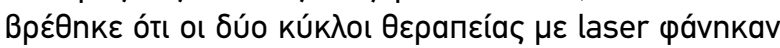

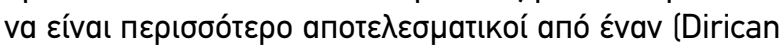
et al 2011).

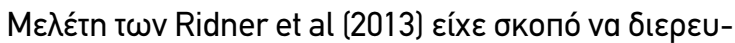

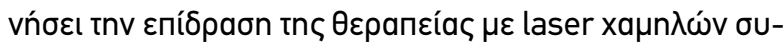

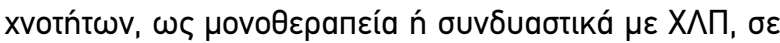

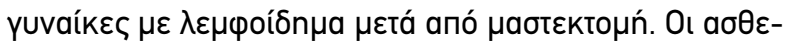




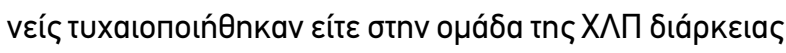

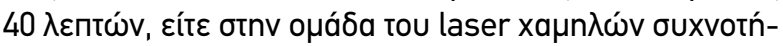

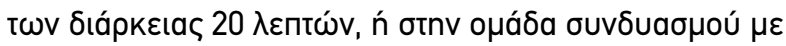

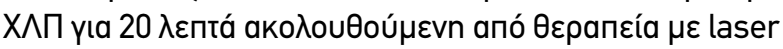

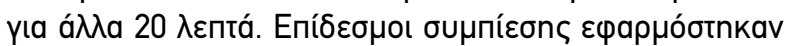

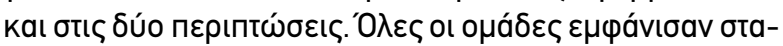

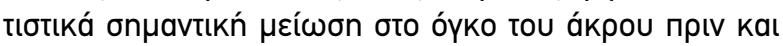

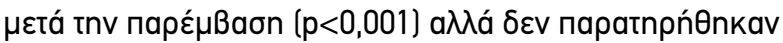

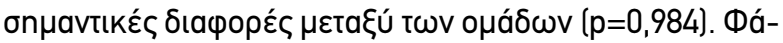

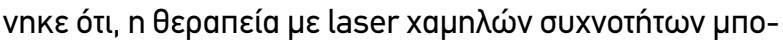

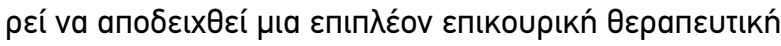

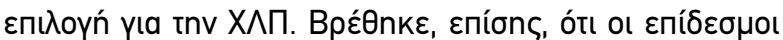

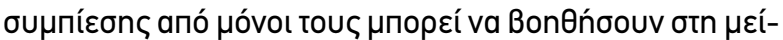
won tou óүkou tou ákpou (Ridner et al 2013).

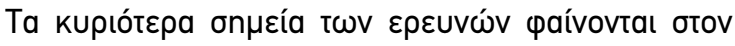
пívaka 6.

\section{EYZHTHEH}

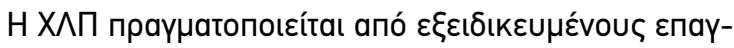

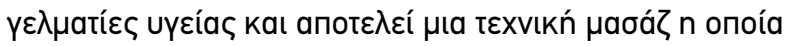

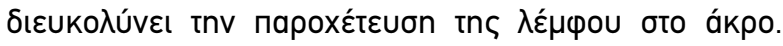

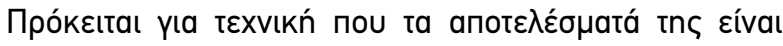

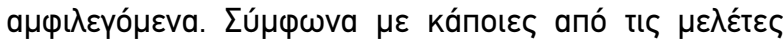

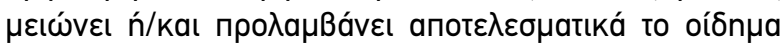

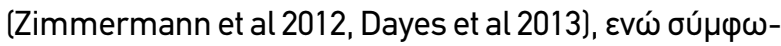

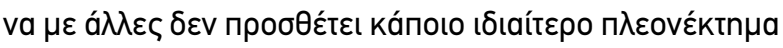
(Gradalski et al 2010, Devoogdt et al 2011, De Oliveira et

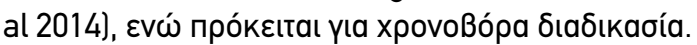

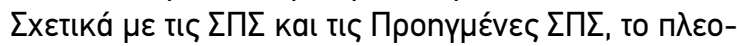

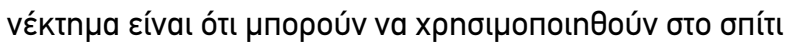

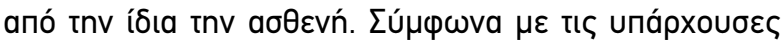

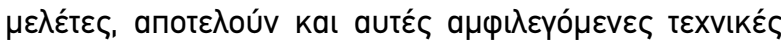

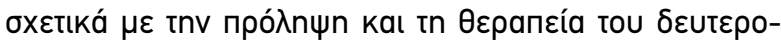

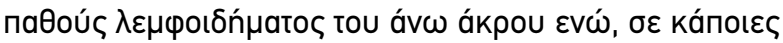

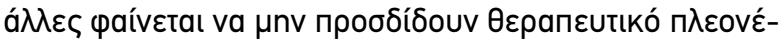

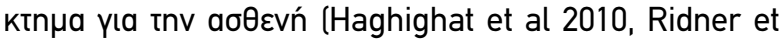

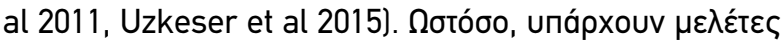

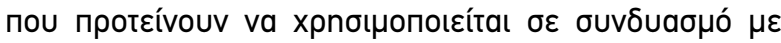

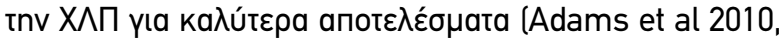
Gurdal et al 2012, Fife et al 2012).

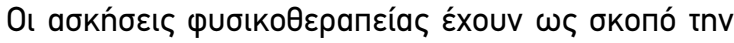

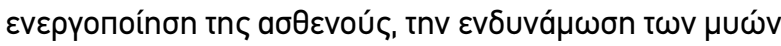

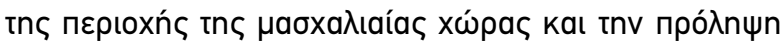

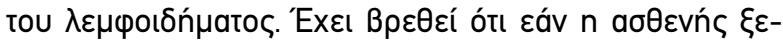

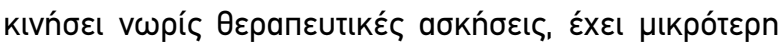

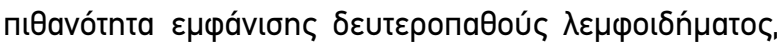

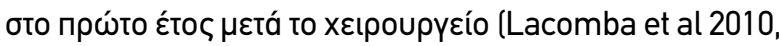

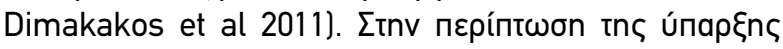

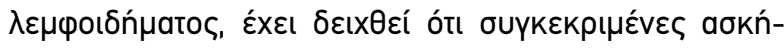

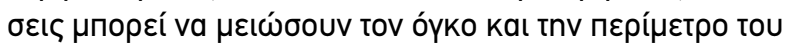

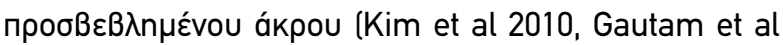

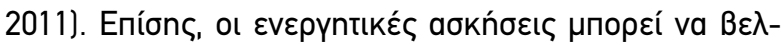

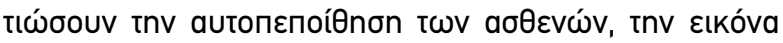

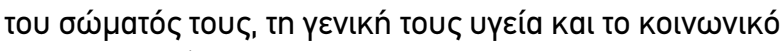
профí $\lambda$ touৎ (Speck et al 2010, Godoy et al 2010, Godoy

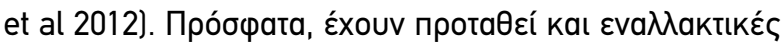

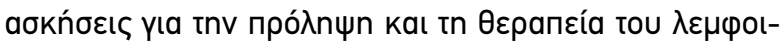

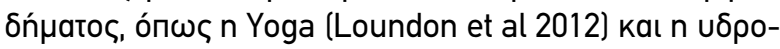

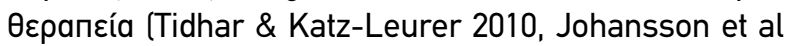

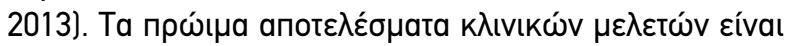

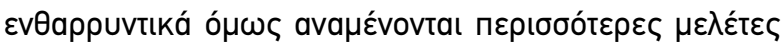

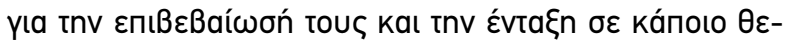

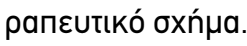

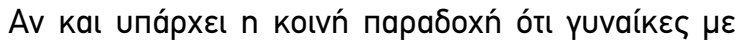

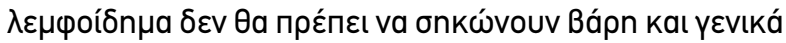

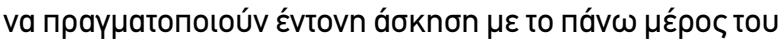

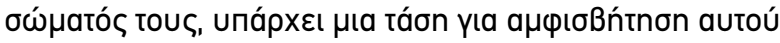

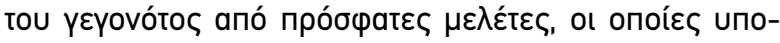

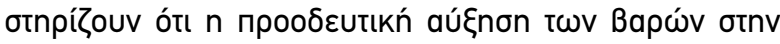

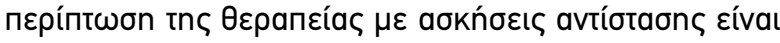

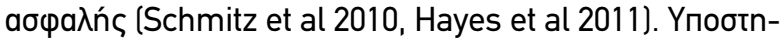

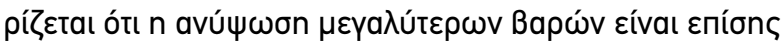

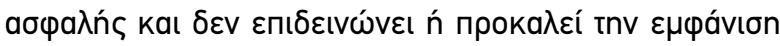

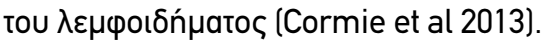

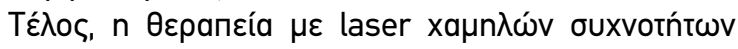

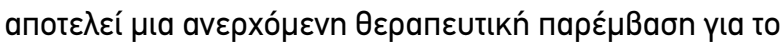

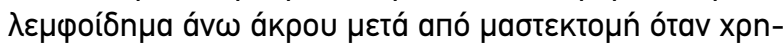

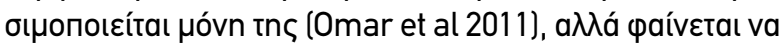

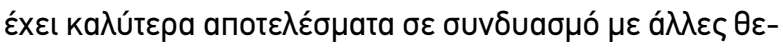

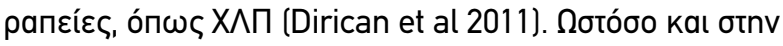

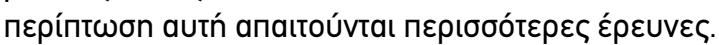

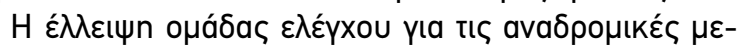

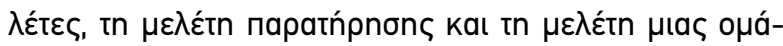

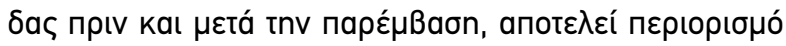

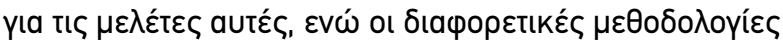

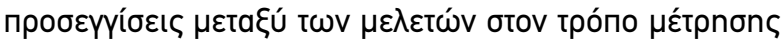

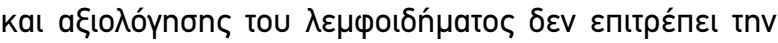

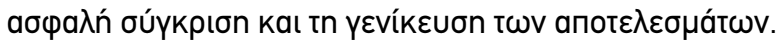

\section{¿YMПEPAгMATA}

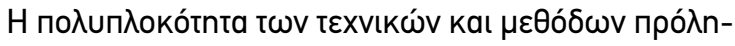

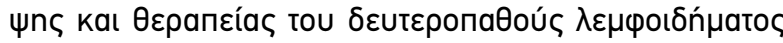

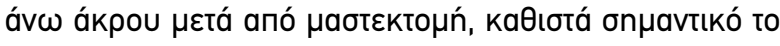

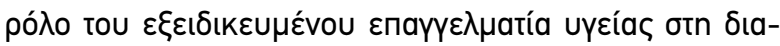

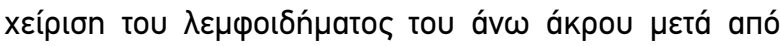

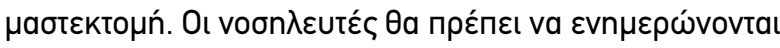

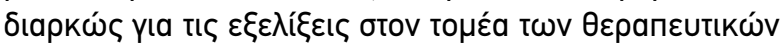

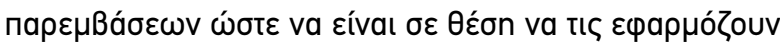

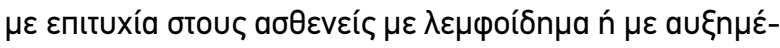

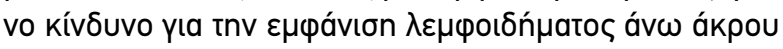

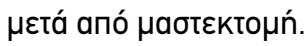

'O

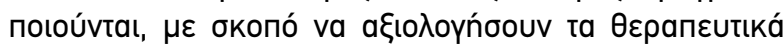

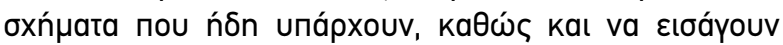

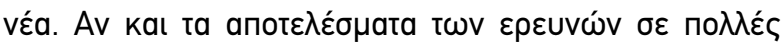

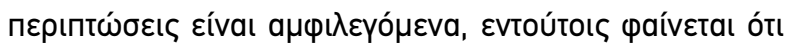




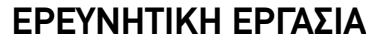

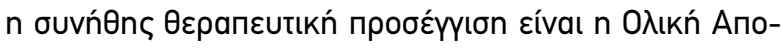

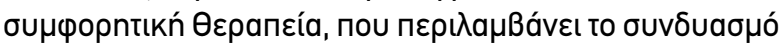

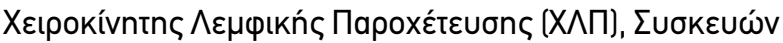

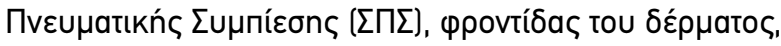

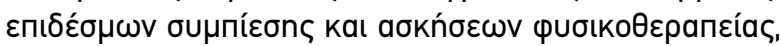

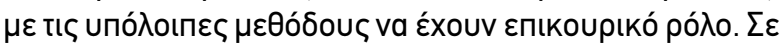

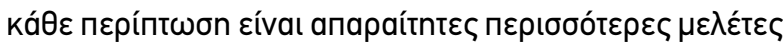

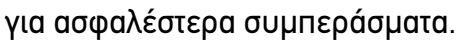

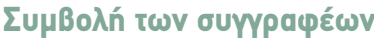

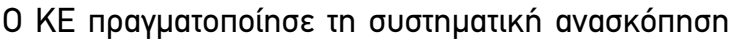

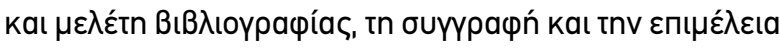

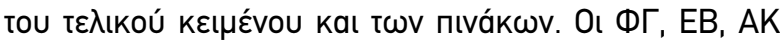

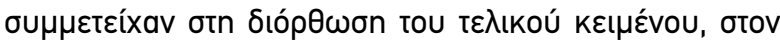

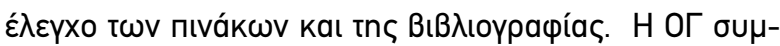

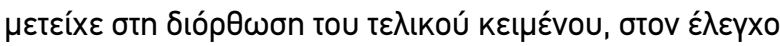

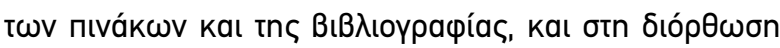

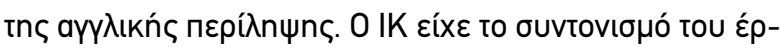

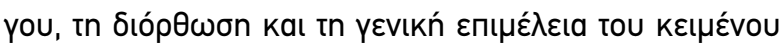

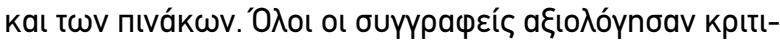

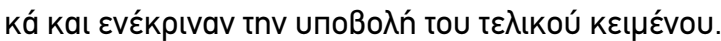

\section{BIBАІОГРАФІА}

Adams K.E., Rasmussen J.C., Darne C., Tan I.C., Aldrich M.B., Marshall M.V., Fife C.E., Maus E.A., Smith L.A., Guilloid R., Hoy S. \& Sevick-Muraca E.M. (2010). Direct evidence, of lymphatic function improvement after advanced pneumatic compression device treatment of lymphedema. Biomedical optics express 1(1):114-125.

Cormie P., Pumpa K., Galvão D.A., Turner E., Spry N., Saunders C. \& Newton R.U. (2013). Is it safe and efficacious for women with lymphedema secondary to breast cancer to lift heavy weights during exercise: a randomised controlled trial. Journal of Cancer Survivorship 7(3):413-424

Dayes I.S., Whelan T.J., Julian J.A., Parpia S., Pritchard K.I., D'Souza D.P., Kligman L., Reise D., Le Blanc L., Mc Neely M.L., Manchul L., Weirnikowski J. \& Levine M.N. (2013). Randomized trial of decongestive lymphatic therapy for the treatment of lymphedema, in women with breast cancer. Journal of clinical oncology $31(30): 3758-3763$.

de Oliveira M.M.F., de Rezende L.F., do Amaral M.T.P., Pinto e Silva M.P., Morais S.S. \& Costa Gurgel M.S. (2014). Manual lymphatic drainage versus exercise in the early postoperative period for breast cancer. Physiotherapy theory and practice 30(6):384-389.

Devoogdt N., Christiaens M.R., Geraerts I., Truijen S., Smeets A., Leunen K., Neven P. \& Van Kampen M. (2011). Effect of manual lymph drainage in addition to guidelines and exercise therapy on arm lymphoedema related to breast cancer: randomised controlled trial. Bmj343:d5326.

Dimakakos E., Kalemikerakis J., Vardaki Z., Fouka G., Antonatos G. \& Krousianotaki K. (2011). Combined decongestive therapy in Greece: an effective treatment of lymphedema. The European Journal of Lymphology and Related Problems 22(63):21 suppl.

Dirican A., Andacoglu O., Johnson R., McGuire K., Mager L. \& Soran A. (2011). The short-term effects of low-level laser therapy in the management of breast-cancer-related lymphedema. Supportive care in cancer 19(5):685-690.

Fife C.E., Davey S., Maus E.A., Guilliod R. Mayrovitz H.N. (2012). A randomized controlled trial comparing two types of pneumatic compression for breast cancer-related lymphedema treatment in the home. Supportive Care in Cancer 20(12):3279-3286.

Gautam A.P., Maiya A.G. \& Vidyasagar M.S. (2011). Effect of homebased exercise program on lymphedema and quality of life in female postmastectomy patients: pre-post intervention study. Journal of Rehabilitation Research Devices 48(10):1261-8.

Godoy M.D.F.G., Oliani A.H. \& de Godoy J.M.P. (2010). Active exercises utilizing a facilitating device in the treatment of lymphedema resulting from breast cancer therapy. GMS Geriatric Medical
Science 8:Doc31.

Godoy M.D.F.G., Pereira M.R., Oliani A.H. \& de Godoy J.M.P. (2012). Synergic effect of compression therapy and controlled active exercises using a facilitating device in the treatment of arm lymphedema. International Journal of Medical Sciences 9(4):280.

Gurdal S.O., Kostanoglu A., Cavdar I., Ozbas A., Cabioglu N., Ozcinar B., Igci A., Muslumanoglu M. \& Ozmen V. (2012). Comparison of intermittent pneumatic compression with manual lymphatic drainage for treatment of breast cancer-related lymphedema. Lymphatic research and biology 10(3):129-135.

Haghighat S., Lotfi-Tokaldany M., Yunesian M., Akbari M.E., Nazemi F. \& Weiss J. (2010). Comparing two treatment methods for post mastectomy lymphedema: complex decongestive therapy alone and in combination with intermittent pneumatic compression. Lymphology 43(1):25.

Hayes S.C., Speck R.M., Reimet E., Stark A. \& Schmitz K.H. (2011). Does the effect of weight lifting on lymphedema following breast cancer differ by diagnostic method: results from a randomized controlled trial. Breast cancer research and treatment 130(1):227.

Hayes S.C., Johansson K., Stout N.L., Prosnitz R., Armer J.M., Gabram S. \& Schmitz K.H. (2012). Upper冈body morbidity after breast cancer. Cancer 118(S8):2237-2249.

Hormes J.M., Bryan C., Lytle L.A., Gross C.R., Ahmed R.L., Troxel A.B. \& Schmitz K.H. (2010). Impact of lymphedema and arm symptoms on quality of life in breast cancer survivors. Lymphology 43(1):113.

Johansson K., Hayes S., Speck R. M. \& Schmitz K.H. (2013). Waterbased exercise for patients with chronic arm lymphedema: a randomized controlled pilot trial. American Journal of Physical Medicine \& Rehabilitation 92(4):312-319.

Kalemikerakis I., Kosma E. \& Dimakakos E. (2012). Effective complete decongestive therapy of upper limb lymphedema in women after mastectomy. Perioperative Nursing-Quarterly scientific, online official journal of GORNA 1 (2 May-August 2012):57-62.

Kim Y.J., Jeong H.J. \& Kim G.C. (2010). Effect of active resistive exercise on breast cancer-related lymphedema: a randomized controlled trial. Archives of physical medicine and rehabilitation 91(12):1844-1848.

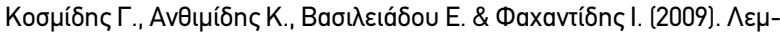

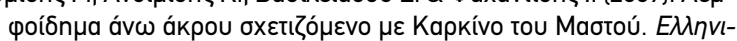

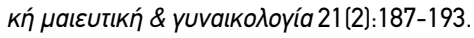

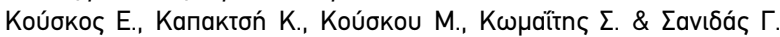

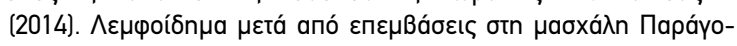

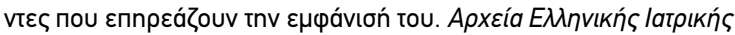
31(1):55-59. 
Lacomba M.T., Sánchez M.J.Y., Goñi Á.Z., Merino D.P., del Moral O.M., Téllez E.C. \& Mogollón E.M. (2010). Effectiveness of early physiotherapy to prevent lymphoedema after surgery for breast cancer: randomised, single blinded, clinical trial. BMJ 340:b5396.

Loudon A., Barnett T., Piller N., Immink M.A., Visentin D. \& Williams A.D. (2012). The effect of yoga on women with secondary arm lymphoedema from breast cancer treatment. $B M C$ complementary and alternative medicine 12(1):66.

Malicka I., Stefańska M., Rudziak M., Jarmoluk P., Pawłowska K., Szczepańska-Gieracha J. \& Woźniewski M. (2011). The influence of Nordic walking exercise on upper extremity strength and the volume of lymphoedema in women following breast cancer treatment. Isokinetics and Exercise Science 19(4):295-304

Moattari M., Jaafari B., Talei A., Piroozi S., Tahmasebi S. \& Zakeri Z. (2012). The effect of combined decongestive therapy and pneumatic compression pump on lymphedema indicators in patients with breast cancer related lymphedema. Iranian Red Crescent Medical Journal 14(4):210.

Ochałek K. \& Gradalski T. (2010). Manual lymph drainage may not be a necessary component in lymphedema treatment. Journal of Pain and Symptom Management 39(5): 1 1-2

Omar M.T.A., Ebid A.A.E.G. \& El Morsy A.M. (2011). Treatment of post-mastectomy lymphedema with laser therapy: double blind placebo control randomized study. Journal of Surgical Research 165(1):82-90.

Paskett E.D., Dean J.A., Oliveri J.M. \& Harrop J.P. (2012). Cancerrelated lymphedema risk factors, diagnosis, treatment, and impact: a review. Journal of Clinical Oncology 30(30):3726-3733.

Reul-Hirche H. (2011). Manual lymph drainage when added to advice and exercise may not be effective in preventing lymphoedema after surgery for breast cancer. Journal of physiotherapy 57(4):258.
Ridner S.H., Murphy B., Deng J., Kidd N., Galford E., Bonner C., Bond S.M. \& Dietrich M.S. (2012). A randomized clinical trial comparing advanced pneumatic truncal, chest, and arm treatment to arm treatment only in self-care of arm lymphedema. Breast cancer research and treatment 131(1):147-158.

Ridner S.H., Poage-Hooper E., Kanar C., Doersam J.K., Bond S.M. \& Dietrich M.S. (2013, July). A pilot randomized trial evaluating low-level laser therapy as an alternative treatment to manual lymphatic drainage for breast cancer-related lymphedema. In Oncology nursing forum (Vol. 40, No. 4). NIH Public Access.

Schmitz K.H., Ahmed R.L., Troxel A.B., Cheville A., Lewis-Grant L., Smith, R., Bryan C.J., Williams-Smith C.T. \& Chittams J. (2010). Weight lifting for women at risk for breast cancer-related lymphedema: a randomized trial. Jama 304(24):2699-2705.

Speck R.M., Gross C.R., Hormes J.M., Ahmed R.L., Lytle L.A., Hwang W.T. \& Schmitz K.H. (2010). Changes in the Body Image and Relationship Scale following a one-year strength training trial for breast cancer survivors with or at risk for lymphedema. Breast cancer research and treatment 121(2):421-430.

Tidhar D. \& Katz-Leurer M. (2010). Aqua lymphatic therapy in women who suffer from breast cancer treatment-related lymphedema: a randomized controlled study. Support Care Cancer 18(3):383-92.

Uzkeser H., Karatay S., Erdemci B., Koc M. \& Senel K. (2015). Efficacy of manual lymphatic drainage and intermittent pneumatic compression pump use in the treatment of lymphedema after mastectomy: a randomized controlled trial. Breast Cancer 22(3):300-307.

Zimmermann A., Wozniewski M., Szklarska A., Lipowicz A. \& Szuba A. (2012). Efficacy of manual lymphatic drainage in preventing secondary lymphedema after breast cancer surgery. Lymphology 45(3): 103 


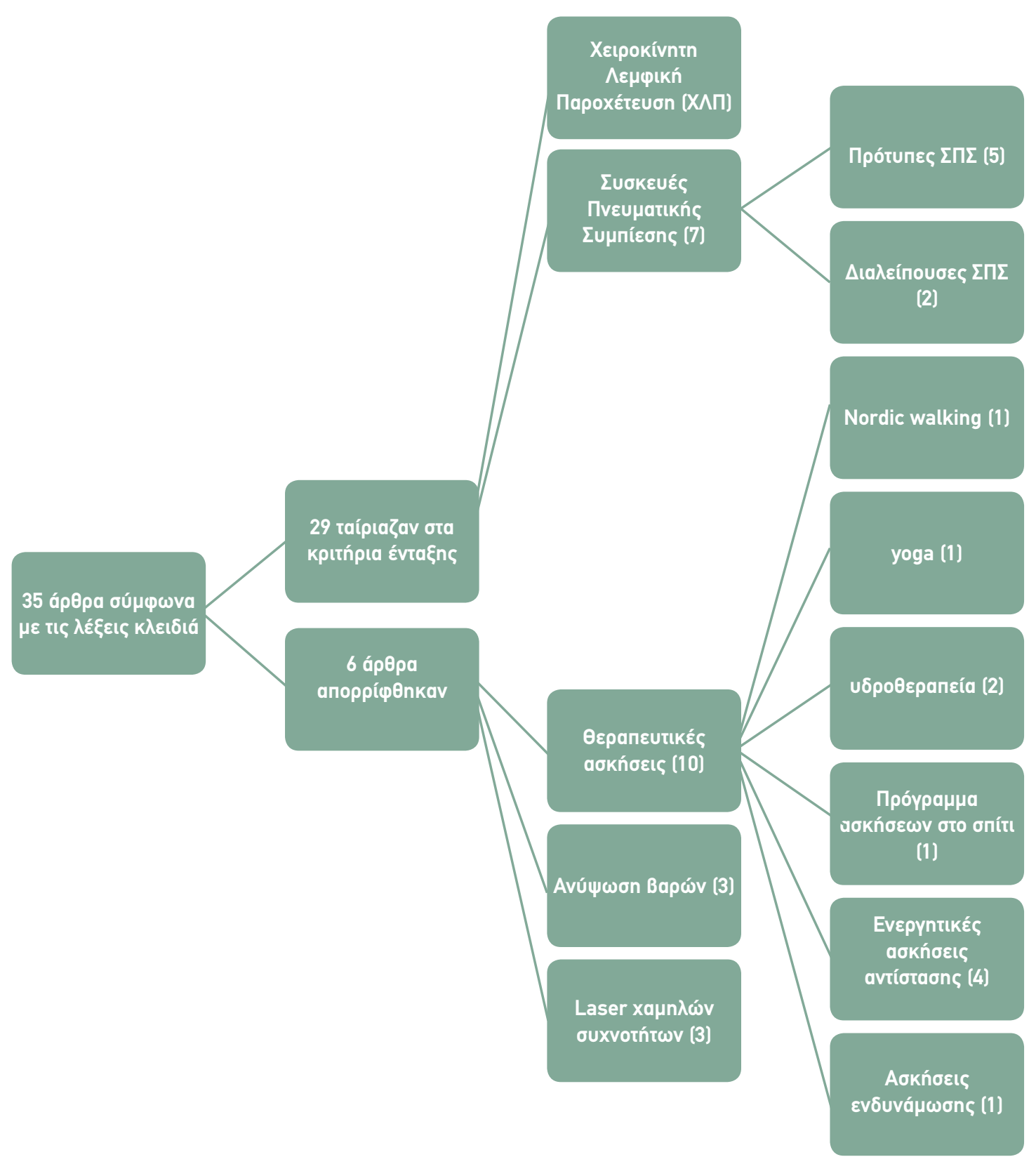




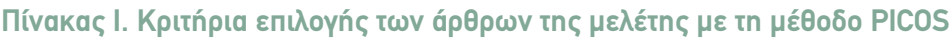

\begin{tabular}{|c|c|}
\hline 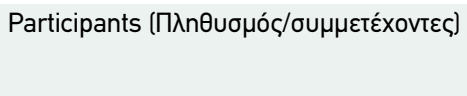 & 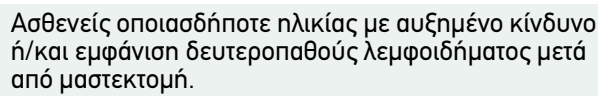 \\
\hline 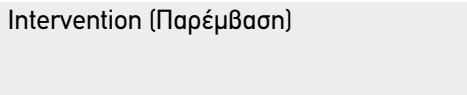 & 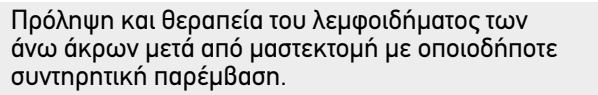 \\
\hline 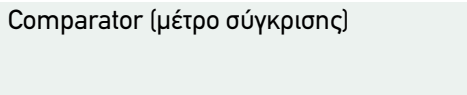 & 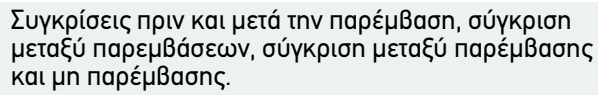 \\
\hline Outcomes (źkßaon) & 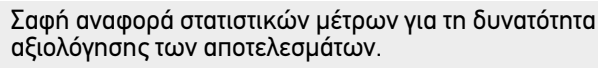 \\
\hline 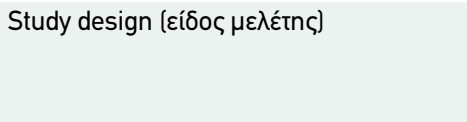 & 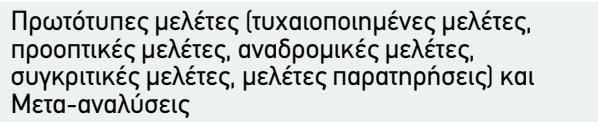 \\
\hline
\end{tabular}

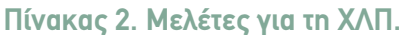

\begin{tabular}{|c|c|c|c|c|}
\hline 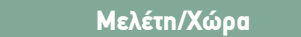 & Asíyua & 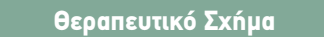 & 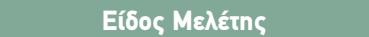 & Euph́pata \\
\hline 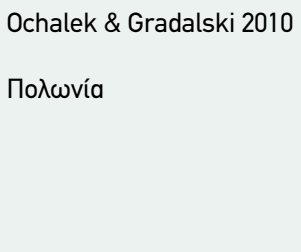 & $\mathrm{n}=50$ & 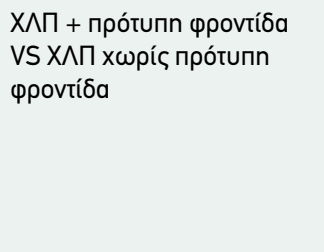 & 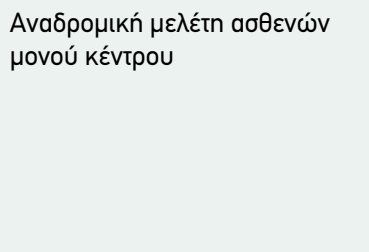 & 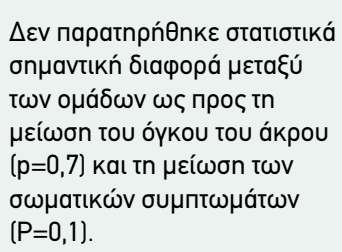 \\
\hline 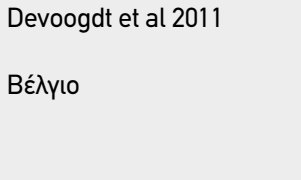 & $n=6$ & 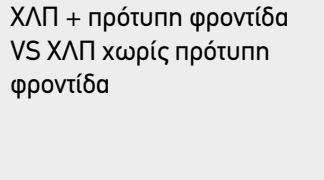 & 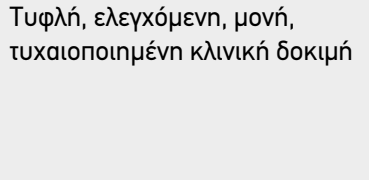 & 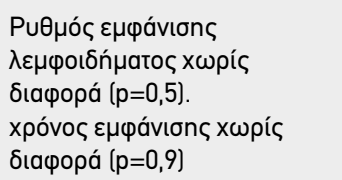 \\
\hline $\begin{array}{l}\text { Reul-Hirche } 2011 \\
\text { Auбтрa入ía }\end{array}$ & $n=160$ & 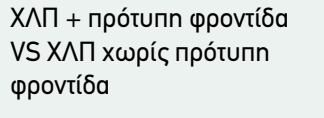 & 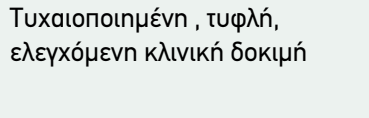 & 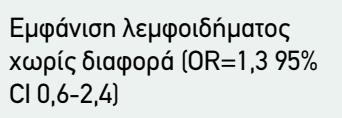 \\
\hline 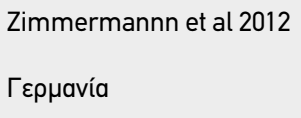 & $\mathrm{n}=67$ & $\begin{array}{l}\text { X^П VS про́тuпn } \\
\text { фчбเкоӨврапві́a }\end{array}$ & 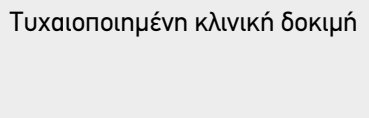 & 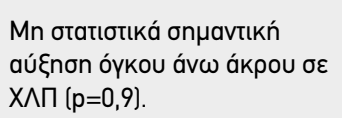 \\
\hline 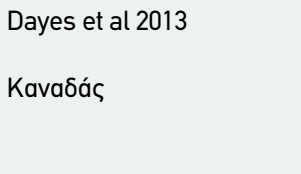 & $\mathrm{N}=95$ & 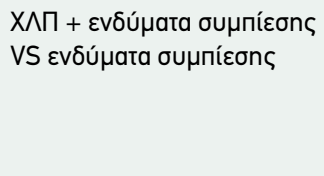 & 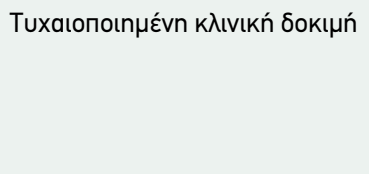 & 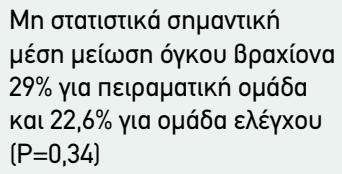 \\
\hline $\begin{array}{l}\text { De Oliveira et al } 2014 \\
\text { Bpaלı入ía }\end{array}$ & $\mathrm{N}=89$ & 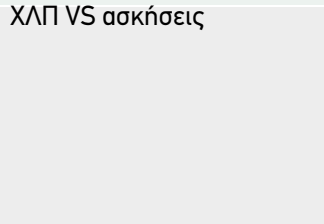 & 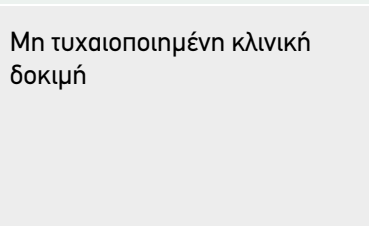 & 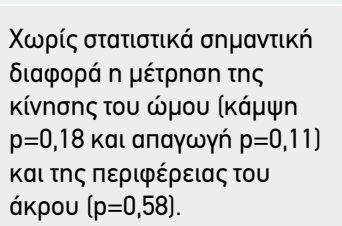 \\
\hline
\end{tabular}




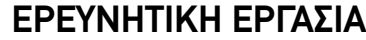

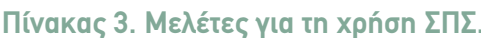

\begin{tabular}{|c|c|c|c|c|}
\hline 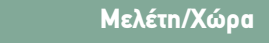 & $\Delta$ вíy & 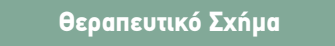 & 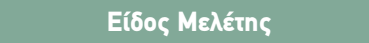 & Euph́pata \\
\hline $\begin{array}{l}\text { Haghighat al } 2010 \\
\text { HПА }\end{array}$ & $n=112$ & 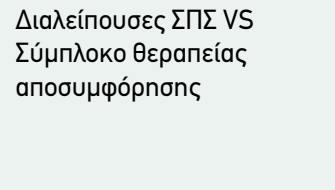 & 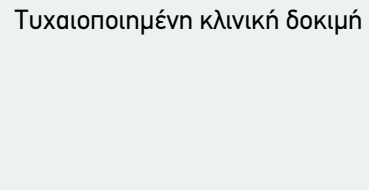 & 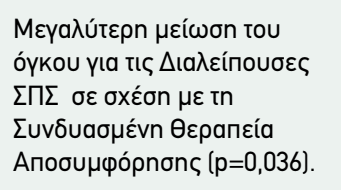 \\
\hline $\begin{array}{l}\text { Adams et al } 2010 \\
\text { HПA }\end{array}$ & $n=6$ & 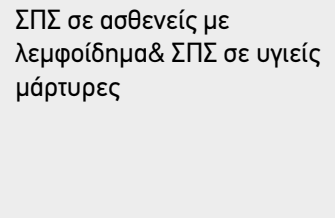 & 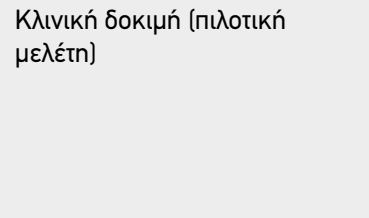 & 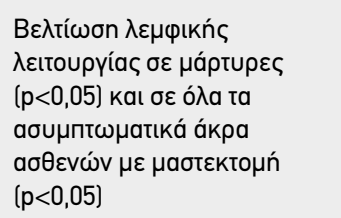 \\
\hline $\begin{array}{l}\text { Ridner et al } 2012 \\
\text { НПА }\end{array}$ & $n=42$ & 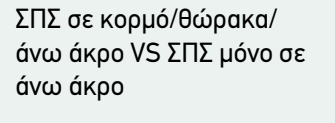 & 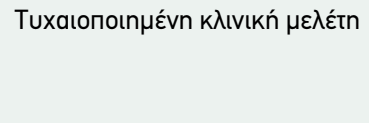 & 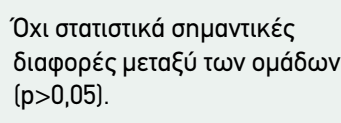 \\
\hline $\begin{array}{l}\text { Fife et al } 2012 \\
\text { НПА }\end{array}$ & $n=36$ & 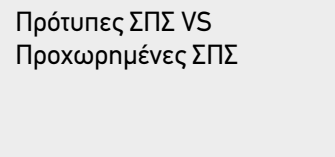 & 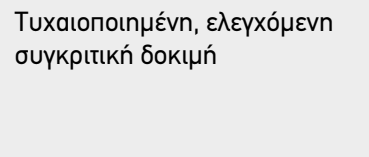 & 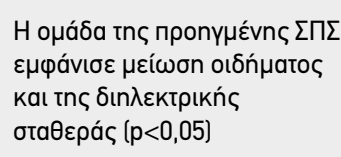 \\
\hline $\begin{array}{l}\text { Gurdal et al } 2012 \\
\text { Toupkía }\end{array}$ & $n=30$ & 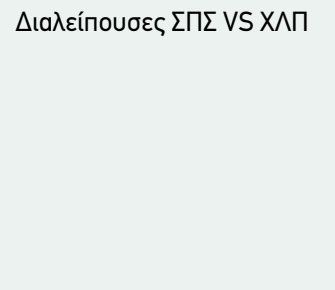 & 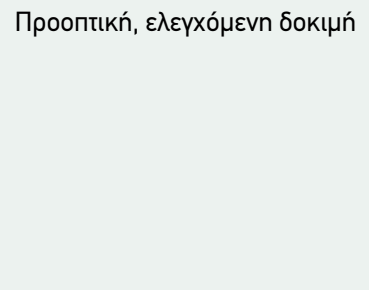 & 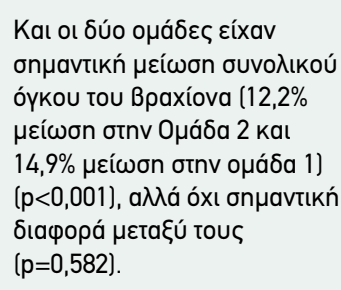 \\
\hline $\begin{array}{l}\text { Moattari et al } 2012 \\
\text { Ipáv }\end{array}$ & $n=21$ & 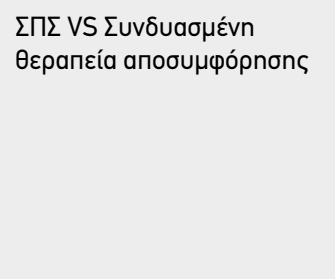 & 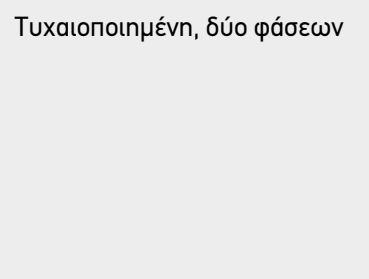 & 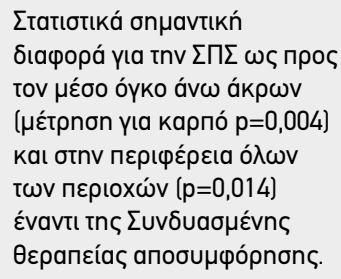 \\
\hline $\begin{array}{l}\text { Uzkeser et al } 2015 \\
\text { Toupkía }\end{array}$ & $n=31$ & $\mathrm{X} \wedge \Pi$ VS $\Sigma \Pi \Sigma$ & 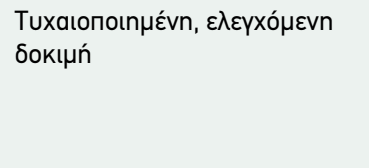 & 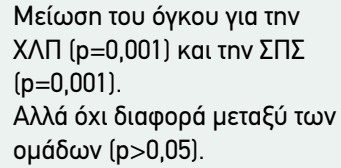 \\
\hline
\end{tabular}




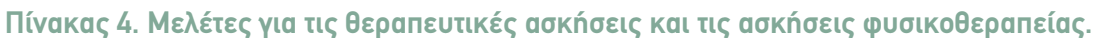

\begin{tabular}{|c|c|c|c|c|}
\hline 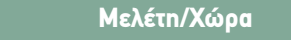 & $\Delta \varepsilon i ́ y \mu$ & 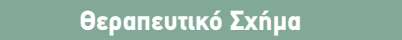 & 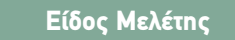 & Eupńpata \\
\hline $\begin{array}{l}\text { Lacomba et al } 2010 \\
\text { Ionavía }\end{array}$ & $n=116$ & 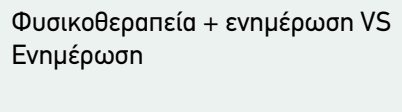 & 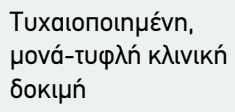 & 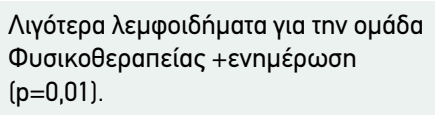 \\
\hline 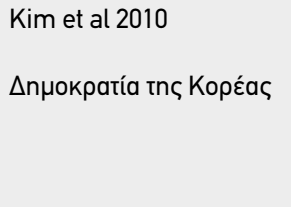 & $n=40$ & 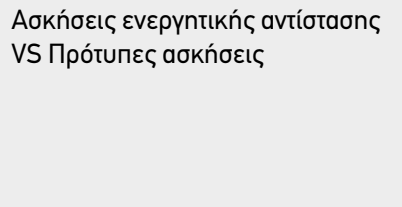 & 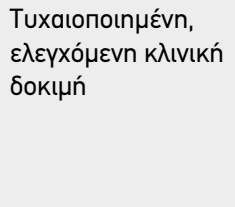 & 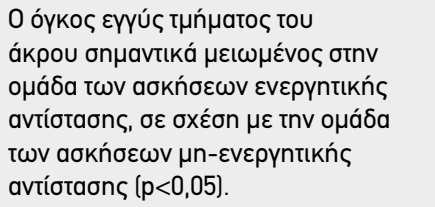 \\
\hline $\begin{array}{l}\text { Speck et al } 2010 \\
\text { HПА }\end{array}$ & $\mathrm{n}=295$ & 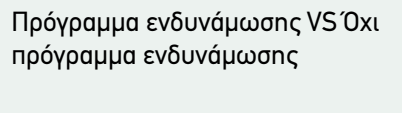 & 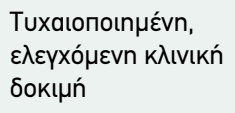 & 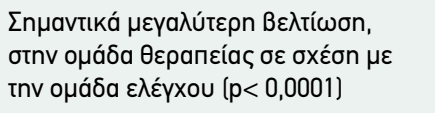 \\
\hline $\begin{array}{l}\text { Tidhar \& Katz-Leurer, } \\
2010 \\
\text { lopań }\end{array}$ & $\mathrm{n}=48$ & 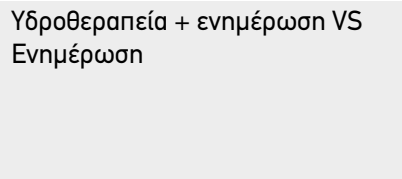 & 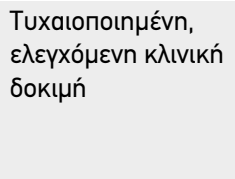 & 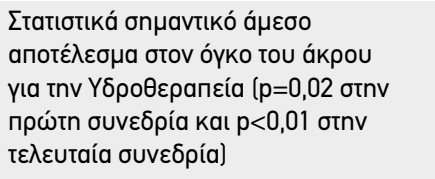 \\
\hline $\begin{array}{l}\text { Godoy et al } 2010 \\
\text { BpaZı入ía }\end{array}$ & $\mathrm{n}=21$ & 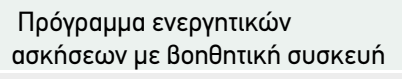 & 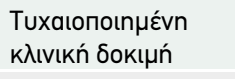 & 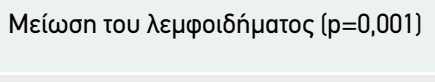 \\
\hline $\begin{array}{l}\text { Gautam et al } 2011 \\
\text { Ivoía }\end{array}$ & $\mathrm{n}=32$ & 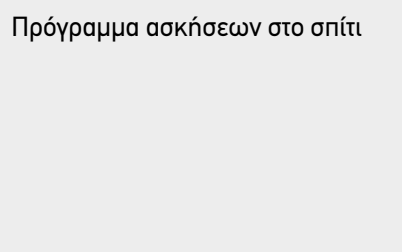 & 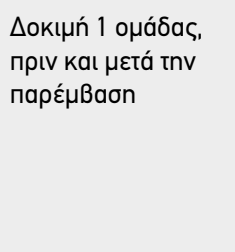 & 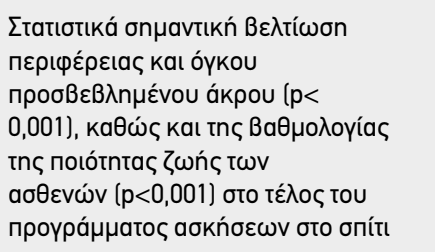 \\
\hline $\begin{array}{l}\text { Godoy et al } 2012 \\
\text { Bpaלı入ía }\end{array}$ & $\mathrm{n}=20$ & 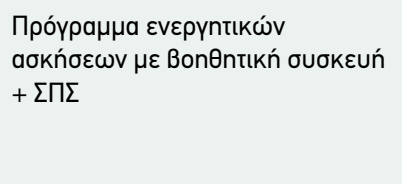 & 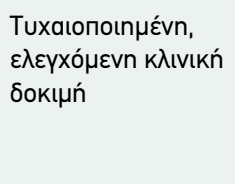 & 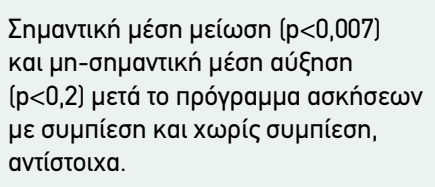 \\
\hline 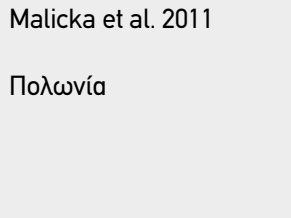 & $\mathrm{n}=38$ & 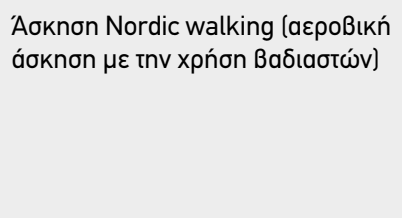 & $\begin{array}{l}\text { ‘epquva } \\
\text { napatńpnons }\end{array}$ & 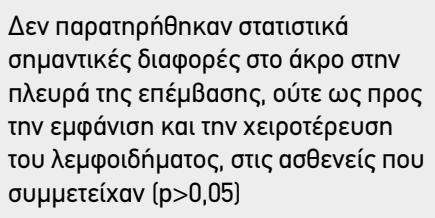 \\
\hline 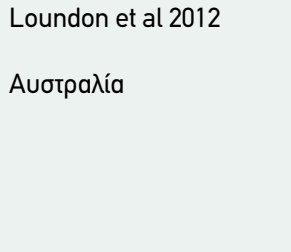 & $n=40$ & Yoga VS Прótunn фpovtíбa & 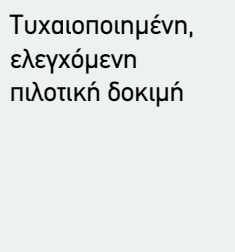 & 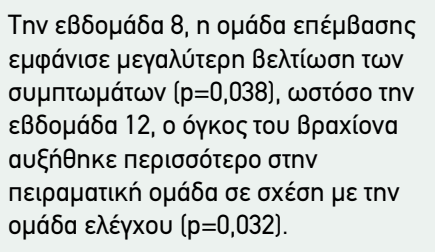 \\
\hline $\begin{array}{l}\text { Johansson et al } 2013 \\
\text { Eounסía }\end{array}$ & $\mathrm{n}=29$ & 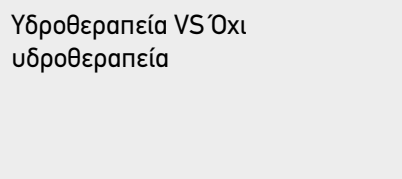 & 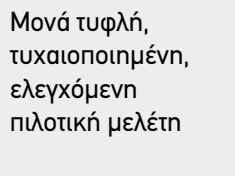 & 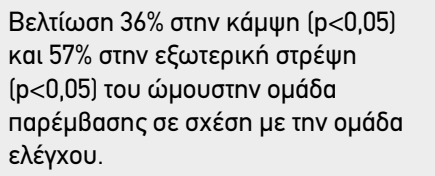 \\
\hline
\end{tabular}




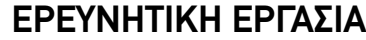

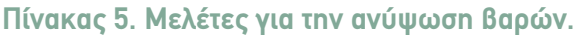

\begin{tabular}{|c|c|c|c|c|}
\hline 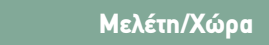 & $\Delta$ вíүра & Өврапєutukó Еxńpa & 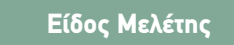 & Euph́para \\
\hline $\begin{array}{l}\text { Schmitz et al } 2010 \\
\text { НПА }\end{array}$ & $n=154$ & 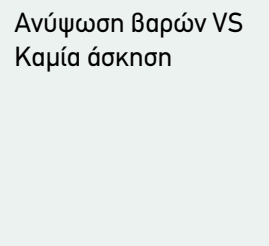 & 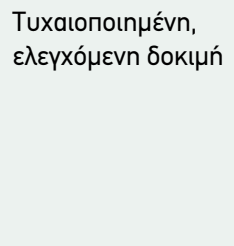 & 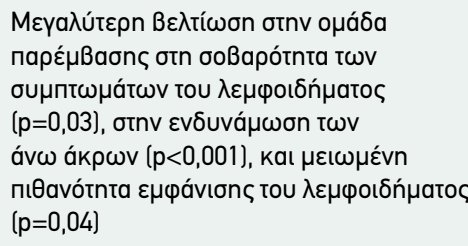 \\
\hline $\begin{array}{l}\text { Hayes et al } 2011 \\
\text { Auotpa入ía }\end{array}$ & $n=295$ & 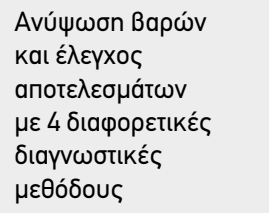 & 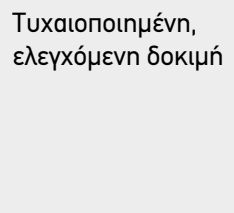 & 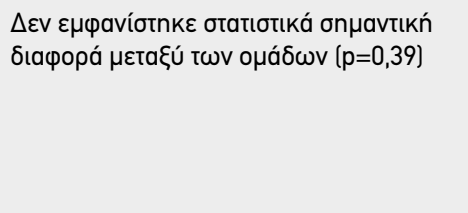 \\
\hline 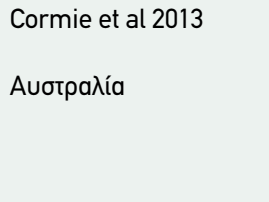 & $\mathrm{n}=62$ & 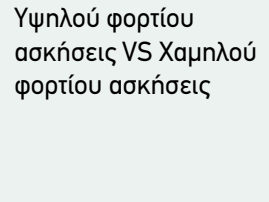 & 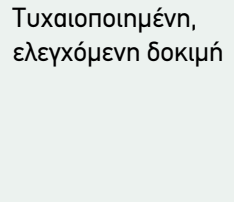 & 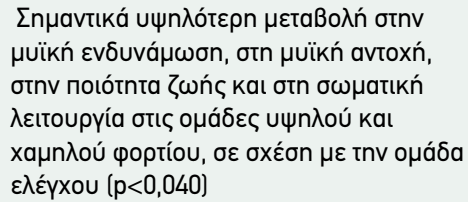 \\
\hline
\end{tabular}

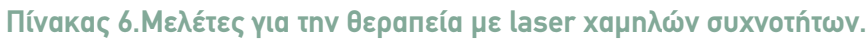

\begin{tabular}{|c|c|c|c|c|}
\hline 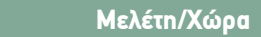 & $\Delta$ síypa & 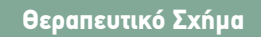 & 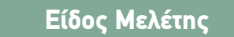 & Euph́pata \\
\hline $\begin{array}{l}\text { Omar et al } 2011 \\
\text { Aípuntos }\end{array}$ & $\mathrm{n}=50$ & Laser VS Placebo & 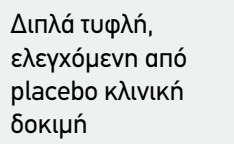 & 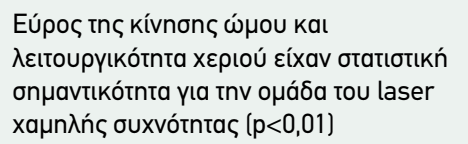 \\
\hline $\begin{array}{l}\text { Dirican et al } 2011 \\
\text { НПА }\end{array}$ & $\mathrm{n}=17$ & $\begin{array}{l}\text { Laser kaı прótunn } \\
\text { фроvтíठ́ }\end{array}$ & 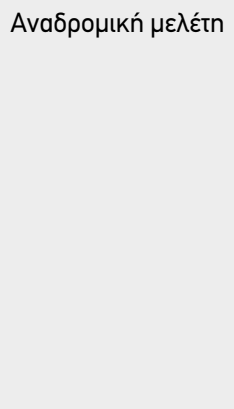 & 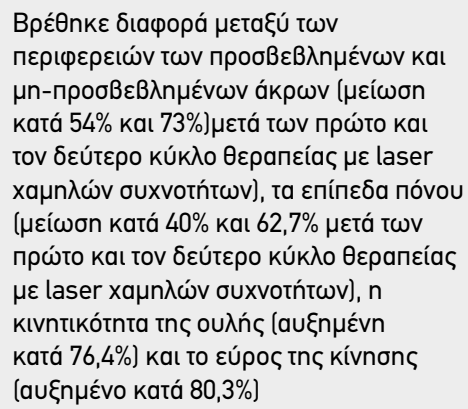 \\
\hline $\begin{array}{l}\text { Ridner et al } 2013 \\
\text { НПА }\end{array}$ & $n=42$ & $\begin{array}{l}\text { ХАП VS laser VS X^П } \\
+ \text { laser }\end{array}$ & 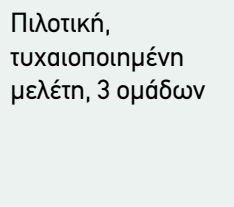 & 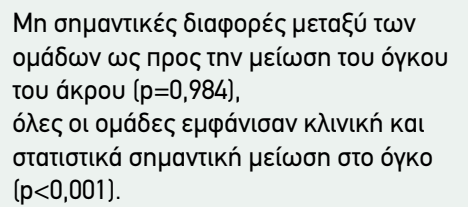 \\
\hline
\end{tabular}




\title{
Prevention and conservative treatment of upper limb secondary lymphedema after mastectomy
}

\author{
Kosmas Emmanouilidis', Georgia Fasoi², Eugenia Vlachou², Ourania Govina², Anna Kavga³, \\ loannis Kalemikerakis ${ }^{3}$ \\ 1. Nurse, MSc, "Metaxa" Specialised Anticancer Hospital of Pireus, Athens \\ 2. Associate Professor, Faculty of Nursing, University of West Attica \\ 3. Lecturer, PhD, Faculty of Nursing, University of West Attica
}

\begin{abstract}
Introduction: Lymphedema is a frequent complication in women after mastectomy. It affects the patient's quality of life and functionality. The aim of this systematic review was to investigate and report appropriate, and evidence-based, guidelines for the prevention and treatment of secondary lymphedema of the upper extremity after mastectomy.

Methodology: Relevant Greek and international literature was searched in the electronic databases PubMed, Cinahl, Sciverse, Scopus and Proquest by using keywords and time restriction.

Results: Thirty-six papers were retrieved, of which 29 fitted the study's inclusion criteria. These papers were addressing five categories of post-surgery interventions: Manual Lymphatic Drainage (MLD), Pneumatic Compression Devices (PCDs), physiotherapy exercises, weight lifting and the use of low frequency laser. MLD is a conventional method with controversial therapeutic effects. Similar are the effects of PCDs, which are predominantly used by patients even at home setting. Physiotherapy exercises are indicated to prevent lymphedema immediately after surgery, while weight lifting does not seem to have a negative effect despite current beliefs. Alternative treatments such as hydrotherapy, yoga, and low laser treatment are under investigation. Standard therapeutic approach includes Complete Decongestive Therapy (CDT), i.e. the combination of Manual Lymph Drainage (MLD), Pneumatic Compression Devices (PCDs), skin care, compression bandages and physiotherapy exercises.

Conclusion: Complete Decongestive Therapy (CDT) is the basis of therapeutic interventions with the remaining methods having a complementary role. However, the effectiveness of interventions is not well documented and more research is needed.
\end{abstract}

Key words: nurse, lymphedema, mastectomy, prevention, treatment. 